\title{
Weak kam theorem on non compact manifolds
}

\author{
Albert FATHI \\ Unité de Mathématiques Pures et Appliqués \\ École Normale Supérieure de Lyon \\ 46, allée d'Italie \\ 69364 Lyon cedex 07, France \\ e-mail: afathi@umpa.ens-lyon.fr \\ Ezequiel MADERNA \\ Instituto de Matemática y Estadística "Prof. Rafael Laguardia" \\ Universidad de la República \\ Herrera y Reissig 565 \\ 1200 Montevideo, Uruguay \\ e-mail: eze@fing.edu.uy
}

\begin{abstract}
In this paper, we consider a time independent $C^{2}$ Hamiltonian, satisfying the usual hypothesis of the classical Calculus of Variations, on a non-compact connected manifold. Using the Lax-Oleinik semigroup, we give a proof of the existence of weak KAM solutions, or viscosity solutions, for the associated Hamilton-Jacobi Equation. This proof works also in presence of symmetries. We also study the role of the amenability of the group of symmetries to understand when the several critical values that can be associated with the Hamiltonian coincide.
\end{abstract}

2000 Mathematics Subject Classification: 49L25, 70H20, 58D19.

Key words: Hamilton Jacobi, non compact manifold, Lax-Oleinik, amenable, critical value, viscosity solution.

\section{Introduction}

Let $M$ be a $C^{\infty}$ connected manifold without boundary. We denote by $T M$ the tangent bundle and by $\pi: T M \rightarrow M$ the canonical projection. A point in $T M$ will be denoted by $(x, v)$ with $x \in M$ and $v \in T_{x} M=\pi^{-1}(x)$. In the same way a point of the cotangent space $T^{*} M$ will be denoted by $(x, p)$ with $x \in M$ and $p \in T_{x}^{*} M$, a linear form on the vector space $T_{x} M$. We will suppose that $g$ is a complete Riemannian metric on $M$. For $v \in T_{x} M$, the norm $\|v\|$ is $g(v, v)^{1 / 2}$. We will denote by $\|\cdot\|$ the dual norm on $T_{x}^{*} M$. 
Except for the appendix, we will suppose that $H: T^{*} M \rightarrow \mathbb{R}$ is a function of class at least $C^{2}$, which satisfies the following three conditions:

(1) (Uniform superlinearity) For every $K \geq 0$, there exists $C^{*}(K) \in \mathbb{R}$ such that

$$
\forall(x, p) \in T^{*} M, H(x, p) \geq K\|p\|-C^{*}(K) ;
$$

(2) (Uniform boundedness) for every $R \geq 0$, we have

$$
A^{*}(R)=\sup \{H(x, p) \mid\|p\| \leq R\}<+\infty ;
$$

(3) $\left(C^{2}\right.$ - strict convexity in the fibers) for every $(x, p) \in T^{*} M$, the second derivative along the fibers $\partial^{2} H / \partial p^{2}(x, p)$ is positive strictly definite.

As usual the function $H$ is called the Hamiltonian.

Theorem 1.1 (Weak KAM). Under the above conditions, there is $c(H) \in \mathbb{R}$, such that the Hamilton-Jacobi equation

$$
H\left(x, d_{x} u\right)=c
$$

admits a global viscosity solution $u: M \rightarrow \mathbb{R}$ for $c=c(H)$ and does not admit any such solution for $c<c(H)$.

Following Mañé we will call $c(H)$ the critical value.

In the case where $M$ is the $n$-dimensional torus $\mathbf{T}^{\mathbf{n}}$, this theorem is due to P.L. Lions, G. Papanicolaou \& S.R.S. Varadhan [12], for $M$ an arbitrary compact connected manifold is due to A. Fathi [9], and when $M$ is a cover of a compact manifold $N$ and $H$ the lift of a function on $T^{*} N$, is due to G. Contreras, R. Iturriaga, G.P. Paternain \& M. Paternain [6]. For an adaptation of the proof in [6] to the general case see the work of Contreras [5] which was done about the same time as the first version of this work. Using a fixed point method, we will give a proof in the spirit of [9]. It has the advantage of working also in the presence of a group of symmetries.

To give situations where this theorem can be applied we remark that if $H: T^{*} M \rightarrow \mathbb{R}$ satisfies the following condition

There exists $\alpha \geq 1, \beta \geq 0$ and $\gamma \geq 1$ such that

$$
\forall(x, p) \in T^{*} M,-\beta+\alpha^{-1}\|p\|^{\gamma} \leq H(x, p) \leq \beta+\alpha\|p\|^{\gamma}
$$

then it satisfies both conditions (1) and (2) above. In particular, if $V: M \rightarrow \mathbb{R}$ is of class $C^{2}$ and bounded, then $H(x, p)=\frac{1}{2}\|p\|^{2}+V(x)$ satisfies condition (1-3). We have

Corollary 1.2 If $V: M \rightarrow \mathbb{R}$ is a bounded $C^{2}$ function on the complete Riemannian manifold $M$, then the Hamilton-Jacobi equation

$$
\frac{1}{2}\left\|d_{x} u\right\|^{2}+V(x)=\sup _{x \in M} V(x)
$$

has a global viscosity solution $u: M \rightarrow \mathbb{R}$. 
Another important class of examples is obtained by lifting the Hamiltonian to coverings of $M$. More precisely, it $r: M^{\prime} \rightarrow M$ is a covering, and $d r^{*}$ : $T^{*} M^{\prime} \rightarrow T^{*} M$ is the induced covering of the cotangent space, we can define the lifted Hamiltonian on $T^{*} M^{\prime}$ as $H^{\prime}=H \circ d r^{*}$. It is clear that $H^{\prime}$ satisfies the hypothesis of the theorem with respect to the lifted metric on $M^{\prime}$. On the other hand, if $u: M \rightarrow \mathbb{R}$ is a solution of the Hamilton-Jacobi equation, its lifting $u^{\prime}=u \circ r$ is a solution of the corresponding equation on $M^{\prime}$ for the same value of the constant $c$. Thus, we have the following inequality concerning the critical values:

$$
c\left(H^{\prime}\right) \leq c(H) .
$$

Thereafter, we will denote, as usual, by $\widetilde{M}$ the universal covering of $M$, and by $\bar{M}$ the Abelian covering, i.e. the covering of $M$ whose group of deck transformations is $H_{1}(M, \mathbb{Z})$. In the same way, the lifted Hamiltonians will be denoted by $\widetilde{H}$ and $\bar{H}$ respectively. We will use the notations $c_{u}(H)$ and $c_{a}(H)$ instead of $c(\widetilde{H})$ and $c(\bar{H})$ for their critical values.

If a group $G$ acts on $M$ by diffeomorphisms, then a canonical action on $T^{*} M$ is defined by the derivatives of these diffeomorphisms. We shall be interested in such actions when in addition they preserve the Hamiltonian. That is to say, the following condition is satisfied

(4) (symmetry) For all $g \in G$, if $x \in M$ and $p \in T_{g(x)} M$ then

$$
H(g(x), p)=H\left(x, p \circ d_{x} g\right) .
$$

Here $g$ denotes at the same time the element of the group and its associated diffeomorphism of $M$.

The study of coverings naturally gives rise to Hamiltonians with symmetries. Indeed, in the above examples, lifted Hamiltonians and lifted solutions are invariant under the group of the automorphisms of the respective coverings. On the other hand, if $G$ is connected and $M$ is compact it can be proved that every global viscosity solution of the Hamilton-Jacobi equation is invariant under $G$ (see [13]).

Theorem 1.3 Under conditions (1-4), there is a constant $c_{\text {inv }}(H) \in \mathbb{R}$ such that the Hamilton-Jacobi equation admits a $G$-invariant global viscosity solution for $c=c_{i n v}(H)$ and does not admit any such solution for $c<c_{i n v}(H)$.

It follows that $c(H) \leq c_{i n v}(H)$. Also note that if the action is proper and discontinuous, the constant $c_{i n v}(H)$ is the critical value of the quotient $M / G$. This is the case when $M$ is the universal covering of a manifold $N$ (not necessarily compact) and $G=\pi_{1}(N)$ its fundamental group.

Among all possible applications of global solutions, we want to stand out their usefulness in the study of the dynamics of the Hamiltonian flow $\phi_{t}^{H}$ of $H$. The description of this flow on the energy levels $H^{-1}(c)$ for which the HamiltonJacobi equation admits a global solution can be expanded, since global solutions give rise to invariant sets in these levels. We will explain now how this method 
becomes much more fruitful by a standard process; for a bounded and closed 1-form $\omega$ on $M$, of class $C^{2}$, define the Hamiltonian $H_{\omega}$ as follows:

$$
H_{\omega}(x, p)=H(x, p+\omega)
$$

It is easy to check that $H_{\omega}$ does also satisfy conditions (1-3), therefore, applying theorem 1.1 to $H_{\omega}$, we obtain an invariant set for the Hamiltonian flow of $H$ in the level set corresponding to the critical value of $H_{\omega}$, i.e. $c\left(H_{\omega}\right)$. Note that this value only depends on the cohomology class of $\omega$, since for any differentiable function $f: M \rightarrow \mathbb{R}$ we have that $u: M \rightarrow \mathbb{R}$ is a global solution for $H$ if and only if $u-f$ is a global solution for $H_{d f}$. Furthermore, this defines a convex and superlinear function on the first real cohomology group $H^{1}(M, \mathbb{R})$. As Mañé pointed out, when $M$ is compact there is an interesting connection between these critical values and Mather's theory on minimizing measures. He showed that

$$
c\left(H_{\omega}\right)=\alpha([\omega]),
$$

where $\alpha: H^{1}(M, \mathbb{R}) \rightarrow \mathbb{R}$ is the convex dual of the Mather's action function on $H_{1}(M, \mathbb{R})$. The strict critical value of $H$ is defined as the smallest value of $H_{\omega}$,

$$
c_{\text {strict }}(H)=\inf \left\{c\left(H_{\omega}\right): \omega \text { closed and bounded 1-form on } M\right\} \text {; }
$$

It is no difficult to see that we always have $c_{a}(H) \leq c_{\text {strict }}(H)$. In [17], G. \& M. Paternain proved, supposing $M$ compact, that the Abelian critical value equals the strict one.

Our next result shows that the energy level corresponding to the universal critical value, i.e. $c_{u}(H)$, can also be treated in this way, provided that the fundamental group verifies an algebraic property, namely the amenability. We recall that

Definition 1.4 A discrete group $G$ is amenable if there is a left (or right) invariant mean on $l^{\infty}(G)$, the space of all bounded functions on $G$.

Finite groups as well as Abelian groups are amenable, and finite extensions of solvable groups are also amenable. On the other hand, if a group contains a free subgroup on two generators then it is not amenable; this is the case of the fundamental group of a compact surface of genus $g \geq 2$. See [18] for the properties of amenable groups. We prove

Theorem 1.5 If $\pi_{1}(M)$ is amenable then $c_{u}(H)=c_{a}(H)=c_{\text {strict }}(H)$.

Finally, observe that in the same work [17], G. \& M. Paternain provide an example in a compact surface of genus 2 such that $c_{u}(H)<c_{a}(H)$, showing that the theorem could be false if the fundamental group of the manifold is not amenable. 


\section{Completeness of the Euler-Lagrange flow}

We now introduce the Lagrangian $L: T M \rightarrow \mathbb{R}$ associated to the Hamiltonian $H$, and prove the completeness of its Euler-Lagrange flow.

We recall that $L: T M \rightarrow \mathbb{R}$ is defined by

$$
\forall(x, v) \in T M, L(x, v)=\max _{p \in T_{x}^{*} M}<p, v>-H(x, p)
$$

Since $H$ is finite everywhere, of class $C^{2}$, superlinear and strictly convex in each fiber $T_{x}^{*} M$, it is well known that $L$ is finite everywhere of class $C^{2}$, strictly convex and superlinear in each fiber $T_{x} M$, and satisfies

$$
\forall(x, p) \in T^{*} M, H(x, p)=\max _{v \in T_{x} M}<p, v>-L(x, v) .
$$

The Legendre transform $\mathcal{L}: T M \rightarrow T^{*} M$ defined by

$$
\mathcal{L}(x, v)=\left(x, \frac{\partial L}{\partial v}(x, v)\right)
$$

is a diffeomorphism of class $C^{1}$. Moreover, we have the equality $\langle p, v\rangle=$ $H(x, p)+L(x, v)$ if and only if $(x, p)=\mathcal{L}(x, v)$.

We will prove a little bit more:

Lemma 2.1 The Lagrangian $L: T M \rightarrow \mathbb{R}$ is of class $C^{2}$ and satisfies

(1) (Uniform superlinearity) For every $K \geq 0$, there exists $C(K) \in \mathbb{R}$ such that

$$
\forall(x, v) \in T M, L(x, v) \geq K\|v\|-C(K) .
$$

(2) (Uniform boundedness) For every $R \geq 0$, we have

$$
A(R)=\sup \{L(x, v) \mid\|v\| \leq R\}<+\infty .
$$

(3) ( $C^{2}$ - strict convexity in the fibers) for every $(x, v) \in T M$, the second derivative along the fibers $\partial^{2} L / \partial v^{2}(x, v)$ is positive strictly definite.

(4) For all $R \geq 0$, we have

$$
\sup \{\|p\| \mid(x, p)=\mathcal{L}(x, v),\|v\| \leq R\}<+\infty,
$$

and also

$$
\sup \{\|v\| \mid(x, p)=\mathcal{L}(x, v),\|p\| \leq R\}<+\infty
$$

Proof. To prove (1), we remark that, for $K \geq 0$ and $(x, v) \in T M$, we have

$$
K\|v\|=\max \left\{<p, v>\mid p \in T_{x}^{*} M,\|p\| \leq K\right\},
$$


from which we obtain

$$
L(x, v) \geq K\|v\|-\max _{\|p\| \leq K} H(x, p) .
$$

We conclude that

$$
L(x, v) \geq K\|v\|-A^{*}(K) .
$$

To prove (2), we remark that, for $K \geq 0$ and $(x, v) \in T M$ with $\|v\| \leq K$, we have

$$
\forall p \in T_{x}^{*} M,<p, v>\leq K\|p\| \leq H(x, p)+C^{*}(K),
$$

from which we obtain $L(x, v) \leq C^{*}(K)$.

As we said before, (3) is well known. To prove (4), suppose $(x, p)=\mathcal{L}(x, v)$, with $\|v\| \leq R$; since $H(x, p)=<p, v>-L(x, v)$, we have

$$
(R+1)\|p\|-C^{*}(R+1) \leq H(x, p)=<p, v>-L(x, v) \leq\|p\| R+C(0),
$$

from which it follows that $\|p\| \leq C^{*}(R+1)+C(0)$. The proof of the other part of (4) is identical.

Corollary 2.2 The Euler-Lagrange flow $\phi_{t}: T M \rightarrow T M$ of $L$ is complete.

Proof. Suppose that $\gamma:(a, b) \rightarrow M$ is an extremal of $L$. The curve $\mathcal{L}(\gamma(s), \dot{\gamma}(s))$ is part of the trajectory of the Hamiltonian flow of $H$, hence $H$ is constant on this curve, we denote this constant by $h_{\gamma}$. By the superlinearity of $H$, setting $\mathcal{L}(\gamma(s), \dot{\gamma}(s))=\left(\gamma(s), p_{\gamma}(s)\right)$, we obtain $\left\|p_{\gamma}(s)\right\| \leq C^{*}(1)+h_{\gamma}$, it follows using part (4) of the lemma above that $\sup \{\|\dot{\gamma}(s)\| \mid s \in(a, b)\}$ is finite. In particular, if for example $a$ is finite then the length of the curve $\gamma$ restricted to $(a, \min \{a+$ $1, b\})$ is finite. Since the Riemannian metric is complete, this together with the boundedness of $\{\|\dot{\gamma}(s)\| \mid s \in(a, b)\}$ is enough to guaranty that $\{(\gamma(s), \dot{\gamma}(s)) \mid$ $s \in(a, \min \{a+1, b\})\}$ is contained in a compact subset of $T M$ and hence that this solution of the Euler-Lagrange differential equation can be extended further if $a$ is finite.

\section{The Lax-Oleinik semigroup}

For a function $u: M \rightarrow[-\infty,+\infty]$ and $t \geq 0$, we define the function

$$
T_{t}^{-} u: M \rightarrow[-\infty,+\infty]
$$

by

$$
T_{t}^{-} u(x)=\inf _{\gamma}\left\{u\left(\gamma(0)+\int_{0}^{t} L(\gamma(s), \dot{\gamma}(s)) d s\right\},\right.
$$

where the infimum is taken on all piecewise $C^{1}$ curves $\gamma:[0, t] \rightarrow M$ with $\gamma(t)=x$.

The following lemma is not difficult to check. 
Lemma 3.1 The family of maps $\left(T_{t}^{-}\right)_{t \geq 0}$ is a non-linear semigroup on the space of functions defined on $M$ with values in $[-\infty,+\infty]$. Moreover, if $k \in \mathbb{R}$ and $u: M \rightarrow[-\infty,+\infty]$ then $T_{t}^{-}(u+k)=k+T_{t}^{-} u$. If $u_{1}, u_{2}: M \rightarrow[-\infty,+\infty]$ are such that $u_{1} \leq u_{2}$ then $T_{t}^{-} u_{1} \leq T_{t}^{-} u_{2}$.

If $c \in \mathbb{R}$, and $U$ is an open subset of $M$, we say that a function $u: U \rightarrow \mathbb{R}$ is dominated by $L+c$ on $U$, and we denote this by $u \prec L+c$ on $U$, if for every piecewise $C^{1}$ curve $\gamma:[a, b] \rightarrow U$, with $a \leq b$ we have

$$
u(\gamma(b))-u(\gamma(a)) \leq \int_{a}^{b} L(\gamma(s), \dot{\gamma}(s)) d s+c(b-a) .
$$

Remark that we do not assume that $u$ is continuous in that definition. In fact continuity of such a $u$ is a consequence of the fact that $u \prec L+c$, see below. The relation $u \prec L+c$ can be thought as an integral inequation, i.e. the one for which the equivalent differential version is written $H\left(x, d_{x} u\right) \leq c$. It is not difficult to see that both conditions agree if we only consider smooth functions. In the sequel $\mathcal{H}(c)$ will denote the set of maps $u: M \rightarrow \mathbb{R}$ with $u \prec L+c$.

Proposition 3.2 (1) If $k \in \mathbb{R}$ and $u: M \rightarrow \mathbb{R}$ then $u \in \mathcal{H}(c)$ if and only if $u+k \in \mathcal{H}(c)$.

(2) Every function in $\mathcal{H}(c)$ is $c+A(1)$-Lipschitzian

$$
\forall x, y \in M,|u(y)-u(x)| \leq(c+A(1)) d(x, y),
$$

where $d$ is the metric associated with the (complete) Riemannian metric on $M$.

(3) If $u: M \rightarrow \mathbb{R}$ is $K$-Lipschitzian then $u \in \mathcal{H}(C(K))$. topology.

(4) The subset $\mathcal{H}(c)$ is convex and closed in $C^{0}(M, \mathbb{R})$ for the compact open

(5) If $c, c^{\prime} \in \mathbb{R}$ are such that $c \leq c^{\prime}$ then $\mathcal{H}(c) \subset \mathcal{H}\left(c^{\prime}\right)$.

(6) If $\mathcal{H}(c) \neq \emptyset$ then $c \geq \sup \{-L(x, 0) \mid x \in M\} \geq-A(0)$.

Proof. Statements (1) and (5) are immediate from the definitions.

Statement (2) follows from the inequality

$$
u(y)-u(x) \leq \int_{0}^{d(x, y)} L(\gamma(s), \dot{\gamma}(s)) d s+c d(x, y) \leq(A(1)+c) d(x, y)
$$

obtained by considering a minimizing geodesic $\gamma:[0, d] \rightarrow M$ with unit speed from $x$ to $y$.

From the uniform superlinearity of $L$, we get that for every piecewise $C^{1}$ curve $\gamma:[a, b] \rightarrow M$

$$
\int_{a}^{b} L(\gamma(s), \dot{\gamma}(s)) d s \geq K d(\gamma(a), \gamma(b))-(b-a) C(K),
$$


hence, for every $K$-Lipschitzian function $u$ on $M$, we have

$$
u(\gamma(b))-u(\gamma(a)) \leq K d(\gamma(a), \gamma(b)) \leq \int_{a}^{b} L(\gamma(s), \dot{\gamma}(s)) d s+C(K)(b-a)
$$

and this proves statement (3).

As to statement (4), note that $\mathcal{H}(c)$ is defined as an intersection of half spaces in $C^{0}(M, \mathbb{R})$, one for each path $\gamma$, and these half spaces are closed for the compact open topology.

To prove (6), observe that if $u \in \mathcal{H}(c)$ and $x \in M$, considering the constant path $\gamma(t) \equiv x$ one obtains

$$
0 \leq \int_{a}^{b} L(\gamma(s), \dot{\gamma}(s)) d s+c(b-a)=(L(x, 0)+c)(b-a)
$$

and then

$$
\forall x \in M, c \geq-L(x, 0)
$$

which implies (6).

Proposition 3.3 (1) If $u: M \rightarrow \mathbb{R}$ then $u \prec L+c$ if and only if $u \leq T_{t}^{-} u+c t$ for all $t \geq 0$. In that case, $u \in C^{0}(M, \mathbb{R})$.

(2) The map $T_{t}^{-}$sends $\mathcal{H}(c)$ into itself.

(3) The map $T^{-}:[0,+\infty) \times \mathcal{H}(c) \rightarrow \mathcal{H}(c),(t, u) \mapsto T_{t}^{-} u$ is continuous for the compact open topology on $\mathcal{H}(c)$.

(4) For each $t>0$ and each $x \in M$, there is a $C^{2}$ curve $\gamma:[0, t] \rightarrow M$ such that $\gamma(t)=x$ and

$$
T_{t}^{-} u(x)=u(\gamma(0))+\int_{0}^{t} L(\gamma(s), \dot{\gamma}(s)) d s,
$$

i.e. the infimum in the definition of $T_{t}^{-} u(x)$ is attained.

Proof. To prove (1), remark that domination of $u$ by $L+c$ is equivalent to

$$
u(x) \leq u(y)+\int_{0}^{t} L(\gamma(s), \dot{\gamma}(s)) d s+c t
$$

for all $x, y$ in $M$ and all piecewise- $C^{1}$ paths $\gamma:[0, t] \rightarrow M$ joining $y$ to $x$. Taking the infimum of the right hand side with $x$ and $t$ fixed, this reads

$$
u(x) \leq T_{t}^{-} u(x)+c t
$$

for all $t \geq 0$ and $x \in M$, i.e. $u \leq T_{t}^{-} u+c t$ for all $t \geq 0$. The converse can be proved along the same (reversed) lines, since the Lagrangian is time independent.

Using Lemma 3.1 and (1) it is not difficult to obtain (2). 
We now prove (3). We already know that all functions in $\mathcal{H}(c)$ are Lipschitzian with Lipschitz constant at most $\theta=c+A(1)$.

Using the constant curve with value $x$, we obtain

$$
T_{t}^{-} u(x) \leq u(x)+t A(0) .
$$

This shows that

$$
T_{t}^{-} u(x)=\inf \left\{u(\gamma(0))+\int_{0}^{t} L(\gamma(s), \dot{\gamma}(s)) d s \mid \gamma \in \mathcal{P}(u, x, t)\right\}
$$

where $\mathcal{P}(u, x, t)$ is the set of piecewise $C^{1}$ curves $\gamma:[0, t] \rightarrow M$ with $\gamma(t)=x$ and $u(\gamma(0))+\int_{0}^{t} L(\gamma(s), \dot{\gamma}(s)) d s \leq u(x)+t A(0)$. In particular, for $\gamma \in \mathcal{P}(u, x, t)$ we have

$$
\int_{0}^{t} L(\gamma(s), \dot{\gamma}(s)) d s \leq t A(0)+u(x)-u(\gamma(0)) .
$$

If $u \in \mathcal{H}(c)$ then its Lipschitz constant is at most $\theta=c+A(1)$, it follows that for $\gamma \in \mathcal{P}(u, x, t)$ we have

$$
\int_{0}^{t} L(\gamma(s), \dot{\gamma}(s)) d s \leq t A(0)+\theta d(x, \gamma(0)) .
$$

Since by the superlinearity of $L$ we have

$$
-C(\theta+1) t+(\theta+1) \text { length }(\gamma) \leq \int_{0}^{t} L(\gamma(s), \dot{\gamma}(s)) d s,
$$

for $\gamma \in \mathcal{P}(u, x, t)$ we conclude that

$$
\text { length }(\gamma) \leq t(A(0)+C(\theta+1)) .
$$

Of course length $(\gamma)$ is the length of $\gamma$ for the Riemannian metric on $M$.

We set $K(c, t)=t(A(0)+C(\theta+1))$. Observe this constant depends only on $c$ and $t$, and neither on $x$ nor on $u$. We define $\mathcal{P}^{\prime}(x, c, t)$ as the set of piecewise $C^{1}$ curves $\gamma:[0, t] \rightarrow M$ with $\gamma(t)=x$ and length $(\gamma) \leq K(c, t)$. Since $\mathcal{P}(u, x, t) \subset$ $\mathcal{P}^{\prime}(x, c, t)$ therefore for every $u \in \mathcal{H}(c)$ we have

$$
T_{t}^{-} u(x)=\inf \left\{u(\gamma(0))+\int_{0}^{t} L(\gamma(s), \dot{\gamma}(s)) d s \mid \gamma \in \mathcal{P}^{\prime}(x, c, t)\right\} .
$$

If $u, v \in \mathcal{H}(c)$ and $\gamma \in \mathcal{P}^{\prime}(x, c, t)$, using that for $\gamma \in \mathcal{P}^{\prime}(x, c, t)$ we have $d(x, \gamma(0))=d(\gamma(t), \gamma(0)) \leq$ length $(\gamma) \leq K(c, t)$, we obtain

$$
\begin{aligned}
T_{t}^{-} v(x) \leq & v(\gamma(0))+\int_{0}^{t} L(\gamma(s), \dot{\gamma}(s)) d s \\
\leq & u(\gamma(0))+\int_{0}^{t} L(\gamma(s), \dot{\gamma}(s)) d s+\mid u(\gamma(0)-v(\gamma(0)) \mid \\
\leq & u(\gamma(0))+\int_{0}^{t} L(\gamma(s), \dot{\gamma}(s)) d s+ \\
& +\sup \{|u(y)-v(y)| \mid d(x, y) \leq K(c, t)\} .
\end{aligned}
$$


Taking the infimum over all $\gamma \in \mathcal{P}^{\prime}(x, c, t)$ we conclude that

$$
T_{t}^{-} v(x) \leq T_{t}^{-} u(x)+\sup \{|u(y)-v(y)| \mid d(x, y) \leq K(c, t)\}
$$

By symmetry this gives

$$
\left|T_{t}^{-} v(x)-T_{t}^{-} u(x)\right| \leq \sup \{|u(y)-v(y)| \mid d(x, y) \leq K(c, t)\} .
$$

If for $A \subset M$ and $u, v: M \rightarrow \mathbb{R}$ we set

$$
\|u-v\|_{A}=\sup _{y \in A}|u(y)-v(y)|,
$$

then we can reformulate de above inequality as

$$
\left\|T_{t}^{-} u-T_{t}^{-} v\right\|_{A} \leq\|u-v\|_{A^{\prime}(c, t)}
$$

where $A^{\prime}(c, t)=\{y \in M \mid \exists x \in A$ with $d(y, x) \leq K(c, t)\}$. Since balls for the Riemannian distance $d$ of finite radius are compact, for $A \subset M$ compact the subset $A^{\prime}(c, t)$ is also compact. This finishes the proof that for each $t \geq 0$, the map $T_{t}^{-}: \mathcal{H}(c) \rightarrow \mathcal{H}(c)$ is continuous for the compact open topology.

To complete the proof of assertion (3), it suffices to show that

$$
\left\|T_{s}^{-} u-T_{t}^{-} u\right\|_{M} \leq|s-t| \max \{A(0), c\}
$$

for all $s, t \geq 0$ and $u \in \mathcal{H}(c)$. Since $\left(T_{t}^{-}\right)_{t \geq 0}$ is a semigroup of maps from $\mathcal{H}(c)$ into itself, we have only to prove it for $s=0$. But the condition $u \in \mathcal{H}(c)$ gives $u \leq T_{t}^{-} u+c t$, and we have seen above that $T_{t}^{-} u \leq u+A(0) t$.

It remains to prove (4). By what we have shown above

$$
T_{t}^{-} u(x)=\inf \left\{u(\gamma(0))+\int_{0}^{t} L(\gamma(s), \dot{\gamma}(s)) d s \mid \gamma \in \mathcal{P}^{\prime}(x, c, t)\right\} .
$$

Since the curves in $\mathcal{P}^{\prime}(x, c, t)$ are all contained in the closed Riemannian ball centered in $x$ and of radius $K(c, t)$, which is compact by the completeness of the metric, Tonelli's theory, see [3], [11] or [14], and the continuity of $u$ then shows that the infimum in the definition of $T_{t}^{-} u(x)$ is attained by a curve which is a minimizer of the action and is therefore $C^{2}$.

\section{Proof of the weak KAM theorem}

Let 1 be the constant function with value 1 in $M$. We denote by $\widehat{C^{0}}(M, \mathbb{R})$ the quotient of the vector space $C^{0}(M, \mathbb{R})$ by its subspace $\mathbb{R} \mathbf{1}$. If $\widehat{q}: C^{0}(M, \mathbb{R}) \rightarrow$ $\widehat{C^{0}}(M, \mathbb{R})$ is the quotient map, by the fact that $T_{t}^{-}(u+k)=k+T_{t}^{-} u$, the semigroup $T_{t}^{-}$induces a semigroup of $\widehat{C^{0}}(M, \mathbb{R})$ that we will denote by $\widehat{T}_{t}^{-}$. 
The topology on $\widehat{C^{0}}(M, \mathbb{R})$ is the quotient of the compact open topology on $C^{0}(M, \mathbb{R})$. With this topology, the space $\widehat{C^{0}}(M, \mathbb{R})$ becomes a locally convex topological vector space.

We will denote by $\widehat{\mathcal{H}}(c)$ the image $\widehat{q}(\mathcal{H}(c))$. The subset $\widehat{\mathcal{H}}(c)$ of $\widehat{C^{0}}(M, \mathbb{R})$ is convex and compact. The convexity of $\widehat{\mathcal{H}}(c)$ follows from that of $\mathcal{H}(c)$. To prove that $\widehat{\mathcal{H}}(c)$ is compact, we introduce $C_{x_{0}}^{0}(M, \mathbb{R})$ the set of continuous functions $M \rightarrow \mathbb{R}$ vanishing at some fixed $x_{0}$. The map $\widehat{q}$ induces a homeomorphism from $C_{x_{0}}^{0}(M, \mathbb{R})$ onto $\widehat{C^{0}}(M, \mathbb{R})$. Since $\mathcal{H}(c)$ is stable by addition of constants, its image $\widehat{\mathcal{H}}(c)$ is also the image under $\widehat{q}$ of the intersection $\mathcal{H}_{x_{0}}(c)=\mathcal{H}(c) \cap C_{x_{0}}^{0}(M, \mathbb{R})$. The subset $\mathcal{H}_{x_{0}}(c)$ is closed in $C^{0}(M, \mathbb{R})$ for the compact open topology, moreover, it consists of functions which all vanish at $x_{0}$ and are $(c+A(1))$-Lipschitzian. It follows from Ascoli's theorem that $\mathcal{H}_{x_{0}}(c)$ is a compact set, hence its image $\widehat{\mathcal{H}}(c)$ by $\widehat{q}$ is also compact. The restriction of $\widehat{q}$ to $\mathcal{H}_{x_{0}}(c)$ induces a homeomorphism onto $\widehat{\mathcal{H}}(c)$.

As a first consequence we conclude that if

$$
c(H)=\inf \{c \in \mathbb{R} \mid \mathcal{H}(c) \neq \emptyset\}
$$

then $\bigcap_{c>c(H)} \widehat{\mathcal{H}}(c) \neq \emptyset$ as the intersection of a decreasing family of compact nonempty subsets. It follows that $\mathcal{H}(c(H))$ is also nonempty because it contains the nonempty subset $\widehat{q}^{-1}\left[\bigcap_{c>c(H)} \widehat{\mathcal{H}}(c)\right]$. map

It is obvious that $\widehat{T}_{t}^{-}(\widehat{q}(u))=\widehat{q}\left[T_{t}^{-} u-T_{t}^{-} u\left(x_{0}\right)\right]$, for $u \in \mathcal{H}_{x_{0}}(c)$. Since the

$$
\begin{aligned}
{[0,+\infty) \times \mathcal{H}_{x_{0}}(c) } & \rightarrow \mathcal{H}_{x_{0}}(c) \\
(t, u) & \mapsto T_{t}^{-} u-T_{t}^{-} u\left(x_{0}\right)
\end{aligned}
$$

is continuous, we conclude that $\widehat{T}_{t}^{-}$induces a continuous semigroup of $\widehat{\mathcal{H}}(c)$ into itself. Since this last subset is a nonempty convex compact subset of the locally convex topological vector space $\widehat{C^{0}}(M, \mathbb{R})$, we can apply the Schauder-Tykhonov theorem, see [7] pages $414-415$, to conclude that $\widehat{T}_{t}^{-}$has a fixed point in $\widehat{\mathcal{H}}(c)$, if $\mathcal{H}(c) \neq \emptyset$, i.e. for all value of $c \geq c(H)$.

If we call $\widehat{q}(u)$ such a fixed point with $u \in \mathcal{H}(C(H))$, we see that for each $t \geq 0$ there exists $c(t) \in \mathbb{R}$ such that $T_{t}^{-} u=u+c(t)$. Using that $T_{t}^{-}$is a semigroup and commutes with the addition of constants, we obtain that $c(s+t)=c(s)+c(t)$ for all $s, t \geq 0$, moreover, the map $t \mapsto c(t)$ is continuous since $t \mapsto T_{t}^{-} u$ is continuous. It follows that $c(t)=c(1) t$. The equality $u=T_{t}^{-} u-c(1) t$ shows that $u \prec L-c(1)$, and hence $-c(1) \geq c(H)$. Since $u \in \mathcal{H}(c(H))$, we must have $u \leq T_{t}^{-} u+c(H) t$, which gives $T_{t}^{-} u-c(1) t \leq T_{t}^{-} u+c(H) t$, for all $t \geq 0$, and $-c(1) \leq c(H)$. We conclude that $-c(1)=c(H)$.

We proved 
Proposition 4.1 If $c(H)=\inf \{c \in \mathbb{R} \mid \mathcal{H}(c) \neq \emptyset\}$, then there exists $u: M \rightarrow \mathbb{R}$ such that $u=T_{t}^{-} u+c(H) t$ for all $t \geq 0$.

\section{Relationship with viscosity solutions}

This section contains results that are well known to specialists. They seem to be more like folklore results that has not been already written down in full generality. We give proofs mainly for the reader who is not an expert in viscosity solutions.

A good first introduction to viscosity solutions of the Hamilton-Jacobi equation is contained in [8]. More thorough treatments can be found in the two books [1] and [2].

If $F: T^{*} N \rightarrow \mathbb{R}$ is a continuous function defined on the cotangent bundle of the smooth manifold $N$, and $c \in \mathbb{R}$, we say that $u: N \rightarrow \mathbb{R}$ is a viscosity subsolution (resp. supersolution) of $F\left(x, d_{x} u\right)=c$, if for each $C^{1}$ function $\phi$ : $N \rightarrow \mathbb{R}$ such that $u-\phi$ admits a maximum (resp. a minimum) at some $x_{0} \in N$, we have $F\left(x_{0}, d_{x_{0}} \phi\right) \leq c$ (resp. $\left.F\left(x_{0}, d_{x_{0}} \phi\right) \geq c\right)$. We say that $u: N \rightarrow \mathbb{R}$ is a viscosity solution, if it is both a subsolution and a supersolution.

If $u: N \rightarrow \mathbb{R}$ is differentiable at some $x_{0}$, and is a viscosity subsolution of $F\left(x, d_{x} u\right)=c$ then necessarily $F\left(x_{0}, d_{x_{0}} u\right) \leq c$, see [1] proposition 4.1 page 62 or [2], lemme 2.5 page 33. Conversely, it is an easy exercise to show that an everywhere differentiable function $u$ which satisfies $F\left(x, d_{x} u\right) \leq c$ at each $x \in N$ is necessarily a viscosity subsolution. The analogous statements are valid for viscosity supersolutions or viscosity solutions.

We will use mainly two sorts of $F$ :

1. The first one is $F=H \mid T^{*} U$, where $H$ is the hamiltonian as given in the introduction, and $U$ is an open subset of $M$. This yields the HamiltonJacobi equation in stationary form $H\left(x, d_{x} u\right)=c$.

2. The second sort is $F(t, s, x, p)=s+H(x, p)$, defined on $T^{*}(I \times U)=I \times \mathbb{R} \times$ $T^{*} U$, where $I$ is an interval of $\mathbb{R}$, and $U, H$ are like in the first case. This yields the Hamilton-Jacobi equation in evolution form $\partial_{t} u+H\left(x, \partial_{x} u\right)=c$.

Here are some properties that we will use.

Proposition 5.1 A continuous function $u: U \rightarrow \mathbb{R}$ is a viscosity subsolution of $H\left(x, d_{x} u\right)=c$ if and only if $u \prec L+c$.

Proof. Suppose $u \prec L+c$. Let $\phi: U \rightarrow \mathbb{R}$ be $C^{1}$, and such that $u-\phi$ admits a maximum at $x_{0}$. This implies $\phi\left(x_{0}\right)-\phi(x) \leq u\left(x_{0}\right)-u(x)$. Fix $v \in T_{x_{0}} M$ and choose $\gamma:(-\delta, \delta) \rightarrow M$, a $C^{1}$ path with $\gamma(0)=x_{0}, \dot{\gamma}(0)=v$. For $t \in(-\delta, 0)$, we obtain $\phi(\gamma(0))-\phi(\gamma(t)) \leq u(\gamma(0))-u(\gamma(t)) \leq \int_{t}^{0} L(\gamma(s), \dot{\gamma}(s)) d s-c t$. Dividing by $-t>0$ yields

$$
\frac{\phi(\gamma(t))-\phi(\gamma(0))}{t} \leq \frac{1}{-t} \int_{t}^{0} L(\gamma(s), \dot{\gamma}(s)) d s+c .
$$


If we let $t \rightarrow 0$, we obtain $d_{x_{0}} \phi(v) \leq L\left(x_{0}, v\right)+c$, hence

$$
H\left(x_{0}, d_{x_{0}} \phi\right)=\sup \left\{d_{x_{0}} \phi(v)-L\left(x_{0}, v\right) \mid v \in T_{x_{0}} M\right\} \leq c .
$$

This shows that $u$ is a viscosity subsolution.

To prove the converse, let $u$ be a viscosity subsolution. First we consider the case where $u$ is differentiable, then $H\left(x, d_{x} u\right) \leq c$ everywhere. If $\gamma:[a, b] \rightarrow$ $U$ is a piecewise $C^{1}$ path, by Fenchel's inequality, we obtain $d_{\gamma(s)} u(\dot{\gamma}(s)) \leq$ $L(\gamma(s), \dot{\gamma}(s))+H\left(\gamma(s), d_{\gamma(s)} u\right) \leq L(\gamma(s), \dot{\gamma}(s))+c$. By integration, we obtain $u(\gamma(b))-u(\gamma(a)) \leq \int_{a}^{b} L(\gamma(s), \dot{\gamma}(s)) d s+c(b-a)$, hence $u \prec L+c$. For a general viscosity subsolution $u$, we first observe that $u$ is locally Lipschitz (as already said above this follows the superlinearity, see [1] proposition 4.1 page 62 or [2], lemme 2.5 page 33). By Rademacher's theorem, $u$ is Lebesgue almost everywhere differentiable, and therefore we must have $H\left(x, d_{x} u\right) \leq c$, for almost every $x \in U$. Since $H(x, p)$ is continuous and convex in $p$, we can apply 8.5 to obtain a sequence of $C^{\infty}$ maps $u_{n}: M \rightarrow \mathbb{R}$ such that $\sup _{x \in U}\left|u_{n}(x)-u(x)\right| \leq 1 / n$ and $H\left(x, d_{x} u_{n}\right) \leq c+1 / n$, we can easily pass to the limit to obtain $u \prec L+c$.

Here is a useful criterion to check that a viscosity subsolution is a solution.

Proposition 5.2 Suppose that the continuous function $u: U \rightarrow \mathbb{R}$ is a viscosity subsolution of $H\left(x, d_{x} u\right)=c$, and that for each $x \in U$, we can find a $C^{1}$ path $\gamma$ : $[a, b] \rightarrow U$, with $a<b, \gamma(b)=x$, and $u(\gamma(b))-u(\gamma(a))=\int_{a}^{b} L(\gamma(s), \dot{\gamma}(s))+c(b-a)$. Then $u$ is a viscosity solution of $H\left(x, d_{x} u\right)=c$.

Proof. We first remark that for a $\gamma:[a, b] \rightarrow U$ such that $u(\gamma(b))-u(\gamma(a))=$ $\int_{a}^{b} L(\gamma(s), \dot{\gamma}(s)) d s+c(b-a)$, then for each $t \in[a, b]$, we also do have $u(\gamma(b))-$ $u(\gamma(t))=\int_{t}^{b} L(\gamma(s), \dot{\gamma}(s)) d s+c(b-t)$. In fact, by the previous proposition 5.1, we know that $u \prec L+c$, hence

$$
\begin{gathered}
u(\gamma(b))-u(\gamma(t)) \leq \int_{t}^{b} L(\gamma(s), \dot{\gamma}(s)) d s+c(b-t) \\
u(\gamma(t))-u(\gamma(a)) \leq \int_{a}^{t} L(\gamma(s), \dot{\gamma}(s)) d s+c(t-a) .
\end{gathered}
$$

If we add these two inequalities we get an equality; hence each one of the two inequalities must be an equality.

Suppose now that $\phi: U \rightarrow \mathbb{R}$ is $C^{1}$, and that $u-\phi$ has a minimum at $x_{0} \in U$. We have $\phi\left(x_{0}\right)-\phi(x) \geq u\left(x_{0}\right)-u(x)$. We pick a $C^{1}$ path $\gamma:[a, b] \rightarrow U$, with $a<b, \gamma(b)=x_{0}$, and such that $u(\gamma(b))-u(\gamma(a))=\int_{a}^{b} L(\gamma(s), \dot{\gamma}(s)) d s+c(b-a)$, then we also do have $u(\gamma(b))-u(\gamma(t))=\int_{t}^{b} L(\gamma(s), \dot{\gamma}(s)) d s+c(b-t)$, for each $t \in[a, b]$. Therefore,

$$
\phi(\gamma(b))-\phi(\gamma(t)) \geq \int_{t}^{b} L(\gamma(s), \dot{\gamma}(s)) d s+c(b-t) .
$$


If, for $t \in(a, b)$, we divide by $b-t$, we obtain

$$
\frac{\phi(\gamma(b))-\phi(\gamma(t))}{b-t} \geq \frac{1}{b-t} \int_{t}^{b} L(\gamma(s), \dot{\gamma}(s)) d s+c .
$$

If we let $t$ tend to $b$, this yields $d_{x_{0}} \phi(\dot{\gamma}(b)) \geq L\left(x_{0}, \dot{\gamma}(b)\right)+c$, hence $H\left(x_{0}, d_{x_{0}} \phi\right) \geq$ $d_{x_{0}} \phi(\dot{\gamma}(b))-L\left(x_{0}, \dot{\gamma}(b)\right) \geq c$.

The proof of the following proposition requires argument very close to the ones given in propositions 5.1 and 5.2.

Proposition 5.3 If $u: M \rightarrow \mathbb{R}$ is Lipschitz, then the function $\widetilde{u}:[0,+\infty) \times M \rightarrow$ $\mathbb{R},(t, x) \mapsto T_{t}^{-} u(x)$, is a viscosity solution on $(0,+\infty) \times M$ of the evolution Hamilton-Jacobi equation $\partial_{t} \widetilde{u}+H\left(x, \partial_{x} \widetilde{u}\right)=0$.

Proof. Since $T_{t}^{-}, t \geq 0$ is a semigroup, for every piecewise $C^{1}$ path $\gamma:[a, b] \rightarrow M$, $0 \leq a<b$, we must have

$$
\widetilde{u}(b, \gamma(b))-\widetilde{u}(a, \gamma(a)) \leq \int_{a}^{b} L(\gamma(s), \dot{\gamma}(s)) d s .
$$

It is then easy to adapt the argument of proposition 5.1 to obtain that $\widetilde{u}$ is a viscosity subsolution of $\partial_{t} \widetilde{u}+H\left(x, \partial_{x} \widetilde{u}\right)=0$ on $(0,+\infty) \times M$.

Since the infimum in the definition of $T_{t}^{-} u(x)$ is achieved for $t>0$, we can find $\gamma:[0, t] \rightarrow M$ such that $\gamma(t)=x$, and

$$
\widetilde{u}(t, \gamma(t))-\widetilde{u}(0, \gamma(0))=\int_{0}^{t} L(\gamma(s), \dot{\gamma}(s)) d s .
$$

Using $(*)$ above, instead of $u \prec L+c$, we can adapt the argument of 5.2 to show that $\widetilde{u}$ is a viscosity supersolution.

We show that the viscosity solutions are precisely the fixed points (modulo constants) of the Lax-Oleinik semigroup. This is also a folklore theorem that would be usually proved through a uniqueness theorem. We provide a different argument using the geometry of our setting.

Theorem 5.4 A continuous function $u: M \rightarrow \mathbb{R}$ is a viscosity solution of $H\left(x, d_{x} u\right)=c$ if and only if it is Lipschitz and satisfies $u=T_{t}^{-} u+c t$, for each $t \geq 0$.

Proof. If $u$ satisfies $u=T_{t}^{-} u+c t$, for each $t \geq 0$, then by proposition 3.3 we know that $u \prec L+c$, hence by proposition 5.1 it is a viscosity subsolution. Moreover, since the infimum in the definition of $T_{1}^{-} u(x)$ is attained for $x \in M$, see part (4) of proposition 3.3, we can find $\gamma:[0,1] \rightarrow M$ with $\gamma(1)=x$, and such that

$$
T_{1}^{-} u(x)=u(\gamma(0))+\int_{0}^{1} L(\gamma(s), \dot{\gamma}(s)) d s .
$$


Since $u(x)=T_{1}^{-} u(x)+c 1$, we obtain

$$
u(\gamma(1))-u(\gamma(0))=\int_{0}^{1} L(\gamma(s), \dot{\gamma}(s)) d s+c 1 .
$$

We can now apply 5.2, to conclude that $u$ is a viscosity solution.

Suppose now that $u$ is a viscosity solution. From 5.1, we know that $u \prec L+c$ and is Lipschitz. We can then define $\widetilde{u}(t, x)=T_{t}^{-} u(x)$. We must show that $\widetilde{u}(t, x)=u(x)-c t$. Since we know that $\widetilde{u}$ is locally Lipschitz it suffices to show that $\partial_{t} \widetilde{u}(t, x)=-c$ at each $(t, x)$ where $\widetilde{u}$ admits a derivative. We fix such a point $(t, x)$ where $\widetilde{u}$ is differentiable. From proposition 5.3 , we know that $\widetilde{u}$ is a viscosity solution of $\partial_{t} \widetilde{u}+H\left(x, \partial_{x} \widetilde{u}\right)=0$. Hence we have to show that $H\left(x, \partial_{x} \widetilde{u}(t, x)\right)=c$. In fact we know already that $H\left(x, \partial_{x} \widetilde{u}(t, x)\right) \leq c$, because $\widetilde{u}(t, \cdot)=T_{t}^{-} u$ which is dominated by $L+c$, like $u$. We now identify the partial derivative $\partial_{x} \widetilde{u}(t, x)$. We choose $\gamma:[0, t] \rightarrow M$ with $\gamma(t)=x$ and $T_{t}^{-} u(x)=u(\gamma(0))+\int_{0}^{t} L(\gamma(s), \dot{\gamma}(s)) d s$. The curve $\gamma$ is a minimizer of the action. In particular, the curve $\gamma$ is a solution of the Euler-Lagrange equation, it follows that the energy $H\left(\gamma(s), \frac{\partial L}{\partial v}(\gamma(s), \dot{\gamma}(s))\right)$ is constant. We want to show that $\left.\partial_{x} \widetilde{u}(t, x)=\frac{\partial L}{\partial v}(\gamma(s), \dot{\gamma}(s))\right)$. Choose a chart $U$ around $x \in M$, pick $\delta>0$ small enough to have $\gamma([t-\delta, t]) \subset U$. Identifying $U$ with an open subset of an Euclidian space, for $y$ close enough to $x$ we can define $\gamma_{y}:[0, t] \rightarrow M$ by $\gamma_{y}(s)=\gamma(s)$, for $s \in[0, t-\delta]$, and $\gamma_{y}(s)=\gamma(s)+\frac{s-(t-\delta)}{\delta}(y-x)$ for $s \in[t-\delta, t]$. Obviously $\gamma_{x}=\gamma, \gamma_{y}(0)=\gamma(0)$, and $\gamma_{y}(t)=y$. It follows that $\widetilde{u}(t, y)=T_{t}^{-} u(y) \leq u(\gamma(0))+\int_{0}^{t} L\left(\gamma_{y}(s), \dot{\gamma}_{y}(s)\right) d s$, with equality at $y=x$. We define the function $\phi$ for $y$ close to $x$ by

$$
\begin{aligned}
\phi(y)= & u(\gamma(0))+\int_{0}^{t} L\left(\gamma_{y}(s), \dot{\gamma}_{y}(s)\right) d s \\
=u(\gamma(0))+\int_{0}^{t-\delta} L(\gamma(s), \dot{\gamma}(s)) d s & \\
& \quad+\int_{t-\delta}^{t} L\left(\gamma(s)+\frac{s-(t-\delta)}{\delta}(y-x), \dot{\gamma}(s)+\frac{y-x}{\delta}\right) d s
\end{aligned}
$$

By the last line, the function $\phi$ is obviously $C^{1}$. Since $\phi(y) \geq \widetilde{u}(t, y)$, with equality at $x$, we must have $d_{x} \phi=\partial_{x} \widetilde{u}(t, x)$. But $\gamma_{x}=\gamma$ is an extremal of the Lagrangian $L$, the first variation formula implies that $d_{x} \phi=\frac{\partial L}{\partial v}(\gamma(t), \dot{\gamma}(t))$, see [11] or any book on Calculus of Variations.

Up to now we have obtained

$$
\begin{aligned}
H\left(x, \partial_{x} \widetilde{u}(t, x)\right) & =H\left(\gamma(t), \frac{\partial L}{\partial v}(\gamma(t), \dot{\gamma}(t))\right) \\
& =H\left(\gamma(0), \frac{\partial L}{\partial v}(\gamma(0), \dot{\gamma}(0))\right)
\end{aligned}
$$

It remains to show that $H\left(\gamma(0), \frac{\partial L}{\partial v}(\gamma(0), \dot{\gamma}(0))\right) \geq c$. 
Choosing a chart around $\gamma(0)$, and making an argument symmetrical to the one given above, we can find for $z$ close to $\gamma(0)$, a path $\gamma^{z}:[0, t] \rightarrow M$ with $\gamma^{z}(0)=z, \gamma^{z}(t)=x, \gamma^{\gamma(0)}=\gamma$, and the action $\psi(z)=\int_{0}^{t} L\left(\gamma^{z}(s), \dot{\gamma}^{z}(s)\right) d s$ is $C^{1}$ with $d_{\gamma(0)} \psi=-\frac{\partial L}{\partial v}(\gamma(0), \dot{\gamma}(0))$. Hence we must prove $H\left(\gamma(0),-d_{\gamma(0)} \psi\right) \geq c$. We have $T_{t}^{-} u(x) \leq u(z)+\psi(z)$ with equality at $z=\gamma(0)$. In particular, $u-(-\psi)$ admits a minimum at $\gamma(0)$. Since $u$ is a viscosity solution of $H\left(x, d_{x} u\right)=c$, we must have $H\left(\gamma(0),-d_{\gamma(0)} \psi\right) \geq c$.

\section{Invariant weak KAM solutions}

This section deals with the Hamilton-Jacobi equation for symmetric Hamiltonians in the sense of condition (4) of the introduction. Theorem 1.3 will be proved in the same way as the weak KAM Theorem; we will show that the space of $G$-invariant functions is preserved by the Lax-Oleinik semigroup, which will enable us to apply once again the fixed point method.

We begin by adopting the following notation: let

$$
\mathcal{I}=\left\{f \in C^{0}(M, \mathbb{R}) \mid f(g(x))=f(x), \forall g \in G\right\}
$$

be the space of $G$-invariant continuous functions on $M$, and for each $c \in \mathbb{R}$ let

$$
\mathcal{H}_{\text {inv }}(c)=\mathcal{H}(c) \cap \mathcal{I}
$$

be the set of the invariant functions which are dominated by $L+c$. It is clear that $\mathcal{H}_{\text {inv }}(c)$ is a closed and convex subset of $\mathcal{H}(c)$. It is also clear that $\widehat{\mathcal{H}}_{\text {inv }}(c)=$ $\widehat{q}\left(\mathcal{H}_{\text {inv }}(c)\right)=\widehat{\mathcal{H}}(c) \cap \widehat{q}(\mathcal{I})$, since $\mathcal{I}$ contains the constant functions. Thus, $\widehat{\mathcal{H}}_{\text {inv }}(c)$ is a compact and convex subset of $\widehat{\mathcal{H}}(c)$. We will also note $\widehat{\mathcal{I}}$ the quotient $\widehat{q}(\mathcal{I})$.

Proposition 6.1 If $H$ verifies conditions (1-4) then we have

(1) $L(x, v)=L\left(g(x), d_{x} g(v)\right)$ for all $(x, v) \in T M$ and $g \in G$,

(2) $T_{t}^{-}(u) \in \mathcal{I}$ for all $t \geq 0$ and $u \in \mathcal{I}$,

(3) $\mathcal{H}_{\text {inv }}(c) \neq \phi$ for all $c \geq C(0)$.

Proof. The last assertion is immediate since constant functions are dominated by $L+C(0) \geq 0$. The first one is a direct consequence of the definition of $L$ and due to the fact that $d_{x} g$ is a linear bijection between $T_{x} M$ and $T_{g(x)} M$ :

$$
\begin{aligned}
L\left(g(x), d_{x} g(v)\right) & =\max _{p \in T_{g(x)}^{*} M}<p, d_{x} g(v)>-H(g(x), p) \\
& =\max _{p \in T_{g(x)}^{*} M}<p \cdot d_{x} g, v>-H\left(x, p . d_{x} g\right) \\
& =\max _{p^{\prime} \in T_{x}^{*} M}<p^{\prime}, v>-H\left(x, p^{\prime}\right)=L(x, v)
\end{aligned}
$$


In order to prove (2), fix a real number $t \geq 0$, a function $u \in \mathcal{I}$, a point $x \in M$ and a symmetry $g \in G$. For any piecewise $C^{1}$ curve $\gamma:[0, t] \rightarrow M$ with $\gamma(t)=x$, we have that $\gamma^{\prime}=g \circ \gamma$ is also piecewise $C^{1}$ and that

$$
u(\gamma(0))+\int_{0}^{t} L(\gamma(s), \dot{\gamma}(s)) d s=u\left(\gamma^{\prime}(0)\right)+\int_{0}^{t} L\left(\gamma^{\prime}(s), \dot{\gamma}^{\prime}(s)\right) d s .
$$

Since $\gamma^{\prime}(t)=g(x)$, it follows that $T_{t}^{-} u(g(x)) \leq T_{t}^{-} u(x)$. If we replace $g$ by $g^{-1}$ and $x$ by $g(x)$, the reversed inequality is obtained.

We now define the invariant critical value for the action of the group $G$ as the constant

$$
c_{\text {inv }}(H)=\inf \left\{c \in \mathbb{R} \mid \mathcal{H}_{\text {inv }}(c) \neq \emptyset\right\} .
$$

By propositions 3.2 and 6.1 , we have that $-A(0) \leq c(H) \leq c_{i n v}(H) \leq C(0)$. Actually, theorem 1.3 is a consequence of the following

Proposition 6.2 There exist a G-invariant function $u: M \rightarrow \mathbb{R}$ such that $u=$ $T_{t}^{-} u+c_{\text {inv }}(H) t$ for all $t \geq 0$.

Proof. We know that $\mathcal{I}$ is stable by $T_{t}^{-}$for all $t \geq=0$. This implies that $\widehat{\mathcal{I}}$ is stable by $\widehat{T}_{t}^{-}$. Therefore $\widehat{\mathcal{H}}_{i n v}(c)$ is also stable by $\widehat{T}_{t}^{-}$for each $c \in \mathbb{R}$. As before, $\widehat{\mathcal{H}}_{i n v}\left(c_{i n v}(H)\right)=\bigcap_{c>c_{i n v}(H)} \widehat{\mathcal{H}}_{i n v}(c)$ is nonempty since it is the intersection of a decreasing family of nonempty compact subsets. Thus, $\widehat{T}_{t}^{-}$induces a continuous semigroup on $\widehat{\mathcal{H}}_{\text {inv }}\left(c_{\text {inv }}(H)\right)$. Applying the Schauder-Tykhonov theorem to the semigroup restricted to the compact and convex set $\widehat{\mathcal{H}}_{i n v}\left(c_{i n v}(H)\right)$ we obtain a fixed point. In other words, there exist an invariant function $u_{i n v}: M \rightarrow \mathbb{R}$ and a continuous function $c: \mathbb{R}^{+} \rightarrow \mathbb{R}$ such that $u_{\text {inv }} \in \mathcal{H}\left(c_{\text {inv }}(H)\right)$ and such that $T_{t}^{-}\left(u_{i n v}\right)=u_{i n v}+c(t)$ for all $t \geq 0$. From the semigroup property we have that $c(t)=c(1) t$ for all $t \geq 0$. We now observe that the equality $u_{i n v}=T_{t}^{-}\left(u_{i n v}\right)-c(1) t$ implies that $u_{i n v} \in \mathcal{H}(-c(1))$ and that $u_{i n v} \notin \mathcal{H}(c)$ for any $c<-c(1)$. We can therefore conclude that $-c(1)=c_{i n v}$.

\section{Equivariant solutions and amenability}

Instead of looking at solutions invariant under the symmetry group $G$, we can look for solutions whose graph of the derivative is invariant under the action of $G$ on $T^{*} M$, or equivalently (assuming $M$ connected) at solutions such that for each $g \in G$, there exists $\rho(g) \in \mathbb{R}$, such that $g^{*} u=u+\rho(g)$, where $g^{*} u(x)=u(g x)$. It is easy to see that $\rho: G \rightarrow \mathbb{R}$ is a group homomorphism. We will denote by $\operatorname{Hom}(G, \mathbb{R})$ the set of group homomorphisms $G \rightarrow \mathbb{R}$. Observe that $\operatorname{Hom}(G, \mathbb{R})$ is naturally an $\mathbb{R}$-vector space for pointwise addition and pointwise multiplication by a scalar. 
Given a homomorphism $\rho: G \rightarrow \mathbb{R}$, we say that $u: M \rightarrow \mathbb{R}$ is $\rho$-equivariant if $g^{*} u=u+\rho(g)$, for every $g \in G$. We set

$$
\mathcal{I}_{\rho}=\left\{u \in C^{0}(M, \mathbb{R}) \mid \forall g \in G, g^{*} u=u+\rho(g)\right\} .
$$

It is obvious that $\mathcal{I}_{\rho}$ is an affine subset of $C^{0}(M, \mathbb{R})$, which is invariant under the addition of a constant. In fact, it is either empty or $\mathcal{I}_{\rho}=u+\mathcal{I}$, for $u \in \mathcal{I}_{\rho}$. In particular, $\mathcal{I}_{0}=\mathcal{I}$.

There are of course cases where $\mathcal{I}_{\rho}$ is empty. For example, if the action of $G$ on $M$ has a relatively compact orbit $G x_{0}$, and $u \in \mathcal{I}_{\rho}$, then for each $g \in G$, $|\rho(g)|=\left|u\left(g x_{0}\right)-u\left(x_{0}\right)\right| \leq 2 \sup _{g^{\prime} \in G}\left|u\left(g^{\prime} x_{0}\right)\right|<+\infty$. In particular $\left|\rho\left(g^{n}\right)\right|$ is bounded independently of $n \geq 1$, therefore $|\rho(g)|=\left|\rho\left(g^{n}\right)\right| / n$ must be 0 .

For $c \in \mathbb{R}, \rho \in \operatorname{Hom}(G, \mathbb{R})$, we set $\mathcal{H}_{\rho}(c)=\mathcal{I}_{\rho} \cap \mathcal{H}(c)$. For $\rho \in \operatorname{Hom}(G, \mathbb{R})$, we define $c(\rho)=\sup \left\{c \in \mathbb{R} \mid \mathcal{H}_{\rho}(c)=\emptyset\right\} \in \mathbb{R} \cup\{+\infty\}$. If $c(\rho)<+\infty$, then $c(\rho)=\inf \left\{c \in \mathbb{R} \mid \mathcal{H}_{\rho}(c) \neq \emptyset\right\}$. The function $c: \operatorname{Hom}(G, \mathbb{R}) \rightarrow \mathbb{R}, \rho \mapsto c(\rho)$ is called the Mather function, compare with [15].

We will say that a homomorphism $\rho: G \rightarrow \mathbb{R}$ is tame, if $c(\rho)<+\infty$. We denote by $\operatorname{Hom}_{\text {tame }}(G, \mathbb{R})$ the set of tame homomorphisms.

Since $\mathcal{I}_{\rho}$ is closed in the compact open topology and invariant by the LaxOleinik semigroup (the proof of proposition 6.1 can be easily adapted), we can generalize the proof of 6.2 to obtain the following theorem:

Theorem 7.1 (Equivariant weak KAM). For each $\rho \in \operatorname{Hom}_{\text {tame }}(G, \mathbb{R})$, we have $\mathcal{H}_{\rho}(c(\rho)) \neq \emptyset$. Moreover, we can find a $\rho$-equivariant viscosity solution $u: M \rightarrow \mathbb{R}$ of $H\left(x, d_{x} u\right)=c(\rho)$, i.e. a viscosity solution which satisfies $g^{*} u=u+\rho(g)$, for each $g \in G$.

Here are some of the properties of tame homomorphisms and of the Mather function.

Proposition 7.2 A homomorphism $\rho: G \rightarrow \mathbb{R}$ is tame if and only if $\mathcal{I}_{\rho}$ contains a Lipschitz function. The set $\operatorname{Hom}_{\text {tame }}(G, \mathbb{R})$ is a vector subspace of $\operatorname{Hom}(G, \mathbb{R})$. The restriction of the Mather function $c: \operatorname{Hom}_{\text {tame }}(G, \mathbb{R}) \rightarrow \mathbb{R}$ is convex. If $\operatorname{Hom}_{\text {tame }}(G, \mathbb{R})$ is finite dimensional (for example if $G$ is finitely generated), then $c$ is superlinear on $\operatorname{Hom}_{\text {tame }}(G, \mathbb{R})$.

Proof. By definition $\operatorname{Hom}_{\text {tame }}(G, \mathbb{R})$ is also the set of $\rho$ such that the intersection $\mathcal{I}_{\rho} \cap\left(\cup_{c \in \mathbb{R}} \mathcal{H}(c)\right)$ is not empty. Since the union $\cup_{c \in \mathbb{R}} \mathcal{H}(c)$ is the set $\operatorname{Lip}(M, \mathbb{R})$, we have

$$
\operatorname{Hom}_{\text {tame }}(G, \mathbb{R})=\left\{\rho \in \operatorname{Hom}(G, \mathbb{R}) \mid \mathcal{I}_{\rho} \cap \operatorname{Lip}(M, \mathbb{R}) \neq \emptyset\right\} .
$$

Since $\operatorname{Lip}(M, \mathbb{R})$ is a vector space and

$$
\lambda_{1} \mathcal{I}_{\rho_{1}}+\lambda_{2} \mathcal{I}_{\rho_{2}} \subset \mathcal{I}_{\lambda_{1} \rho_{1}+\lambda_{2} \rho_{2}}
$$

it follows that $\operatorname{Hom}_{\text {tame }}(G, \mathbb{R})$ is a vector subspace of $\operatorname{Hom}(G, \mathbb{R})$. 
If $\lambda_{1}, \lambda_{2} \geq 0$, with $\lambda_{1}+\lambda_{2}=1$, then $\lambda_{1} \mathcal{H}\left(c_{1}\right)+\lambda_{2} \mathcal{H}\left(c_{2}\right) \subset \mathcal{H}\left(\lambda_{1} c_{1}+\lambda_{2} c_{2}\right)$. Together with the inclusion above, this gives convexity.

We prove the superlinearity when $\operatorname{Hom}_{\text {tame }}(G, \mathbb{R})$ is finite dimensional. For each $g \in G$ we consider the linear form $\widehat{g}: \operatorname{Hom}_{\text {tame }}(G, \mathbb{R}) \rightarrow \mathbb{R}, \rho \mapsto \rho(g)$. The family of linear forms generates a vector subspace which is contained in the dual space of $\operatorname{Hom}_{\text {tame }}(G, \mathbb{R})$ and is therefore finite dimensional, hence we can find $g_{1}, \ldots, g_{k} \in G$ such that any other $\widehat{g}$ is a linear combination of $\widehat{g}_{1}, \ldots, \widehat{g}_{k}$. In particular, if $\rho \in \operatorname{Hom}_{\text {tame }}(G, \mathbb{R})$, it follows that $\rho\left(g_{1}\right)=\cdots=\rho\left(g_{2}\right)=0$ implies $\rho=0$. We can therefore use $\|\rho\|=\max _{i=1}^{k}\left|\rho\left(g_{i}\right)\right|$ as a norm on the finite dimensional vector space $\operatorname{Hom}_{\text {tame }}(G, \mathbb{R})$. If $\rho$ is given, let $u: M \rightarrow \mathbb{R}$ be such that $u \in \mathcal{I}_{\rho}$ and $u \prec L+c(\rho)$. We have $n \rho\left(g_{i}\right)=\rho\left(g_{i}^{n}\right)=u\left(g_{i}^{n} x_{0}\right)-u\left(x_{0}\right)$, for $n \in \mathbb{N}$, $i=1, \ldots, k$, and $x_{0}$ some fixed point in $M$. Let us choose a path $\gamma_{i, n}:[0,1] \rightarrow M$ with $\gamma_{i, n}(0)=x_{0}$ and $\gamma_{i, n}(1)=g_{i}^{n} x_{0}$, using $u \prec L+c(\rho)$, we obtain

$$
n \rho\left(g_{i}\right)=u\left(g_{i}^{n} x_{0}\right)-u\left(x_{0}\right) \leq \int_{0}^{1} L\left(\gamma_{i, n}(s), \dot{\gamma}_{i, n}(s)\right) d s+c(\rho) .
$$

The constant $A_{i, n}=\int_{0}^{1} L\left(\gamma_{i, n}(s), \dot{\gamma}_{i, n}(s)\right) d s$ is independent of $\rho$. Arguing in the same way as above with $g_{i}^{-1}$ instead of $g_{i}$, we obtain a constant $A_{i, n}^{\prime}$ independent of $\rho$ and such that

$$
-n \rho\left(g_{i}\right)=u\left(g_{i}^{-n} x_{0}\right)-u\left(x_{0}\right) \leq A_{i, n}^{\prime}+c(\rho) .
$$

If we set $A_{n}=\max \left(A_{1, n}, \ldots, A_{k, n}, A_{1, n}^{\prime}, \ldots, A_{k, n}^{\prime}\right)$, we have obtained a constant $A_{n} \in \mathbb{R}$ depending on $n$ but not on $\rho$, and such that

$$
n\|\rho\|=n \max \left(\rho\left(g_{1}\right), \ldots, \rho\left(g_{k}\right),-\rho\left(g_{1}\right), \ldots,-\rho\left(g_{k}\right)\right) \leq A_{n}+c(\rho) .
$$

Since $n \in \mathbb{N}$ is an arbitrary integer, this proves the superlinearity.

We set

$$
c_{G, \min }(H)=\inf \{c(\rho) \mid \rho \in \operatorname{Hom}(G, \mathbb{R})\}=\inf \left\{c(\rho) \mid \rho \in \operatorname{Hom}_{\text {tame }}(G, \mathbb{R})\right\}
$$

Lemma 7.3 There exists $\rho \in \operatorname{Hom}_{\text {tame }}(G, \mathbb{R})$ such that $c_{G, \min }(H)=c(\rho)$.

Proof. Of course, when $\operatorname{Hom}_{\text {tame }}(G, \mathbb{R})$ is finite dimensional, this follows from the superlinearity of the function $c$.

For the general case, let us pick a decreasing sequence $c\left(\rho_{n}\right) \in \mathbb{R}$, with $\rho_{n} \in \operatorname{Hom}_{\text {tame }}(G, \mathbb{R})$, and $c_{G, \min }(H)=\lim _{n \rightarrow \infty} c\left(\rho_{n}\right)$. For each $n \in \mathbb{N}$, we can find $u_{n} \in \mathcal{H}_{\rho_{n}}\left(c\left(\rho_{n}\right)\right)$. The functions $u_{n}$ form an equi-Lipschitzian set of functions, because they are all contained in $\mathcal{H}\left(c\left(\rho_{0}\right)\right)$. Subtracting a constant from each $u_{n}$, and extracting a subsequence if necessary, we can assume that $u_{n}$ converges uniformly on compact subsets to a function $u: M \rightarrow \mathbb{R}$. Since $u_{n}$ is in the closed set $\mathcal{H}\left(c\left(\rho_{n_{0}}\right)\right)$, for $n \geq n_{0}$, we must have $u \in \mathcal{H}\left(c\left(\rho_{n_{0}}\right)\right)$, for each $n_{0} \in \mathbb{N}$, 
and hence $u \in \mathcal{H}\left(c_{G, \min }(H)\right)$, by $c_{G, \min }(H)=\lim _{n \rightarrow \infty} c\left(\rho_{n}\right)$. Since for $x \in M$, we have $\rho_{n}(g)=u_{n}(g x)-u_{n}(x)$, we conclude that $\rho_{n}$ converges (pointwise) to $\rho \in \operatorname{Hom}(G, \mathbb{R})$, and $u \in \mathcal{I}_{\rho}$. It follows that $c(\rho) \leq c_{G, \min }(H)$. But the reverse inequality follows from the definition of $c_{G, \min }(H)$.

We will now consider the case where $G$ is amenable. Let us recall that this means that there exists (for example) a right invariant mean on $l^{\infty}(G)$, the space of real valued and bounded functions on $G$, i.e. a linear form $m: l^{\infty}(G) \rightarrow \mathbb{R}$ such that

1. $m(c)=c$, for a constant function $c$,

2. $m\left(\varphi_{1}\right) \geq m\left(\varphi_{2}\right)$, if $\varphi_{1}(g) \geq \varphi_{2}(g)$ for every $g \in G$, and

3. $m\left(g_{*} \varphi\right)=m(\varphi)$, where for $g \in G$ and for $\varphi: G \rightarrow \mathbb{R}$, the function $g_{*} \varphi$ is defined by $g_{*} \varphi\left(g^{\prime}\right)=\varphi\left(g^{\prime} g\right)$, for each $g^{\prime} \in G$.

Theorem 7.4 If $G$ is an amenable group then $c_{G, \min }(H)=c(H)$.

Proof. Since obviously $c(H) \leq c_{G, \min }(H)$, it suffices to show that there exists $u \in \mathcal{H}(c(H))$ such that $g^{*} u-u$ is constant for each $g \in G$. We choose $v \in$ $\mathcal{H}(c(H))$, and $x_{0} \in M$. For $x \in M$, consider the map $\varphi_{x}: G \rightarrow \mathbb{R}, g \mapsto$ $v(g x)-v\left(g x_{0}\right)$. We, of course, endow $M$ with the distance $d$ coming from the Riemannian metric. The map $v$ is Lipschitzian for $d$, let $\theta$ be its Lipschitz constant. By lemma 7.5 below, there is a constant $K$ such that $d(g x, g y) \leq K d(x, y)$, for $g \in G, x, y \in M$. In particular, we have $\left|\varphi_{x}(g)\right|=\left|v(g x)-v\left(g x_{0}\right)\right| \leq \theta K d\left(x, x_{0}\right)$, hence $\varphi_{x} \in l^{\infty}(G)$. Therefore we can define $u: M \rightarrow \mathbb{R}$ by $u(x)=m\left(\varphi_{x}\right)$. Let us compute $u(g x)-u(x)$. First $\varphi_{g x}$ is the function $h \in G \mapsto v(h g x)-v\left(h x_{0}\right)=$ $v(h g x)-v\left(h g x_{0}\right)+\left[v\left(h g x_{0}\right)-v\left(h x_{0}\right)\right]=\varphi_{x}(h g)+\varphi_{g x_{0}}(h)$, hence $\varphi_{g x}=g_{*} \varphi_{x}+\varphi_{g x_{0}}$. Therefore, by the properties of $m$, we obtain $m\left(\varphi_{g x}\right)=m\left(\varphi_{x}\right)+m\left(\varphi_{g x_{0}}\right)$. This yields $u(g x)-u(x)=m\left(\varphi_{g x_{0}}\right)$, but the left hand side is clearly independent of $x$. It remains to show that $u \in \mathcal{H}(c(H))$. Let $\gamma:[a, b] \rightarrow M$ be a piecewise $C^{1}$ path. Since $L\left(g x, d_{x} g(v)\right)=L(x, v)$, the path $t \mapsto g \gamma(t)$ has the same action as $\gamma$, therefore using that $v \prec L+c(H)$, we obtain

$$
v(g \gamma(b))-v(g \gamma(a)) \leq \int_{a}^{b} L(\gamma(s), \dot{\gamma}(s)) d s+c(H)(b-a) .
$$

If we add and subtract the quantity $v\left(g x_{0}\right)$ to the left hand side, we obtain

$$
\varphi_{\gamma(b)}(g)-\varphi_{\gamma(a)}(g) \leq \int_{a}^{b} L(\gamma(s), \dot{\gamma}(s)) d s+c(H)(b-a) .
$$

Using the properties of $m$, and taking into account that the right hand side is a constant, we get

$$
u(\gamma(b))-u(\gamma(a)) \leq \int_{a}^{b} L(\gamma(s), \dot{\gamma}(s)) d s+c(H)(b-a) .
$$


It remains to prove the following lemma that was used in the proof of last theorem. Note that this lemma does not use the amenability assumption.

Lemma 7.5 There is a constant $K$ depending only on $H$, such that every diffeomorphism $f: M \rightarrow M$ preserving $H$ is $K$-Lipschitzian for the distance obtained from the Riemannian metric.

Proof. It suffices to show that $d_{x} f(v) \leq C(1)+A(1)$, for $v \in T_{x} M$, with $\|v\|_{x} \leq 1$, where $C(1)$ and $A(1)$ are given by lemma 2.1. In fact, using $L\left(f(x), d_{x} f(v)\right)=$ $L(x, v)$, which follows from the invariance of $H$ by $f$, if $\|v\|_{x} \leq 1$, we obtain

$$
-C(1)+\left\|d_{x} f(v)\right\|_{f(x)} \leq L\left(f(x), d_{x} f(v)\right)=L(x, v) \leq A(1) .
$$

Proof of theorem 1.5. We will consider the lift $\widetilde{H}$ of $H$ to the universal cover $\widetilde{M}$. The fundamental group $\pi_{1}(M)$ acts by deck transformations on $\widetilde{M}$. These deck transformations are symmetries of $\widetilde{H}$. The abelianization of the group $\pi_{1}(M)$ is nothing but $H_{1}(M, \mathbb{Z})$, therefore $\operatorname{Hom}\left(\pi_{1}(M), \mathbb{R}\right)$ is nothing but $H^{1}(M, \mathbb{R})$, the first de Rham cohomology group of $M$. The identification can be given in the following way, if $\omega$ is a smooth closed 1-form on $M$, its lift $\widetilde{\omega}$ to the simply connected manifold $\widetilde{M}$ is exact therefore we can find a smooth function $\widetilde{f}_{\omega}: \widetilde{M} \rightarrow \mathbb{R}$ such that $d \widetilde{f}_{\omega}=\widetilde{\omega}$. Since $M$ is assumed connected $\widetilde{f}_{\omega}$ is well defined up to a constant. Moreover, since $\widetilde{\omega}$ is invariant under deck transformation $g^{*} \widetilde{f}_{\omega}-\widetilde{f}_{\omega}$ is a constant which we denote by $\rho_{\omega}(g)$. Obviously $\rho_{\omega} \in \operatorname{Hom}\left(\pi_{1}(M), \mathbb{R}\right)$. The reader will easily check that $\rho_{\omega}=\rho_{\omega+d u}$, if $u: M \rightarrow \mathbb{R}$ is a smooth function. Since every $\rho_{\omega}$-equivariant function is the sum of $\tilde{f}_{\omega}$ and a function invariant under deck transformations (hence the lift of a function on $M$ ), it follows that solving $\widetilde{H}\left(\widetilde{x}, d_{\widetilde{x}} \widetilde{v}\right) \leq c$ almost everywhere, with $\widetilde{v}: \widetilde{M} \rightarrow \mathbb{R} \rho$-equivariant, is equivalent to solving $\bar{H}\left(x, \omega_{x}+d_{x} u\right) \leq c$ almost everywhere, with $u: M \rightarrow \mathbb{R}$. Therefore $c\left(H_{\omega}\right)=c\left(\rho_{\omega}\right)$. Theorem 1.5 now follows easily from theorem 7.4 .

To finish this section let us give a criterion to verify that a homomorphism is tame.

Proposition 7.6 Let $\rho \in \operatorname{Hom}(G, \mathbb{R})$. The following statements are equivalent

1. the homomorphism $\rho$ is tame,

2. there exists $x_{0} \in M$ and a constant $C_{0}$, such that $\rho(g) \leq C_{0} d\left(g x_{0}, x_{0}\right)$ for $g \in G$,

3. there exists a constant $C$ such that $|\rho(g)| \leq C d(g x, x)$ for $g \in G, x \in M$, where $d$ is the distance obtained from the Riemannian metric. 
Proof. Obviously (3) implies (2). We first show that (1) implies (3). Assuming (1), we can find $u: M \rightarrow \mathbb{R} \rho$-equivariant with $u \in \mathcal{H}(c)$, for some $c \in \mathbb{R}$, therefore $u$ is Lipschitzian. If $C$ is a Lipschitz constant for $u$, we thus have $|\rho(g)|=|u(g x)-u(x)| \leq C d(g x, x)$.

Assume now (2). For each $g \in G$, we define $u_{g}: M \rightarrow \mathbb{R}$ by $u_{g}(x)=$ $C_{0} d\left(g x, x_{0}\right)-\rho(g)$. Since $\left|d\left(g x, x_{0}\right)-d\left(g y, x_{0}\right)\right| \leq d(g x, g y)$, for $g \in G, x, y \in$ $M$, lemma 7.5 shows that all functions $u_{g}$ are equi-Lipschitzian with constant $K C_{0}$. Since $u_{g}\left(x_{0}\right)=C_{0} d\left(g x, x_{0}\right)-\rho(g) \geq 0$, it follows that $u=\inf _{g \in G} u_{g}$ is a function with finite values which is also Lipschitzian with constant $K C_{0}$. Moreover, for $g \in G, x \in M$, we have $u(x)=\inf _{g^{\prime} \in G} C_{0} d\left(g^{\prime} g x, x_{0}\right)-\rho\left(g^{\prime} g\right)=$ $\inf _{g^{\prime} \in G} C_{0} d\left(g^{\prime} g x, x_{0}\right)-\rho\left(g^{\prime}\right)-\rho(g)=-\rho(g)+u(g x)$, hence $u$ is Lipschitz and $\rho$-equivariant.

\section{Appendix}

In this appendix, we will denote by $M$ a smooth metrizable manifold, not necessarily connected. We will suppose that $M$ is endowed with some auxiliary Riemannian metric, not necessarily complete, we will denote by $\|\cdot\|$ the associated norm on any fiber $T_{x} M$ or $T_{x}^{*} M$. We will denote by $\pi_{*}: T^{*} M \rightarrow M$ the canonical projection.

If $f: M \rightarrow \mathbb{R}$ is a locally Lipschitz function, we will denote by $\operatorname{dom}(d f)$ the set of points $x \in M$ where the derivative $d_{x} f$ exists. By Rademacher's theorem $\operatorname{dom}(d f)$ is of full (Lebesgue) measure in $M$.

The goal of this appendix is to prove the following theorem, and obtain some of its consequences.

Theorem 8.1 Let $M$ be a smooth metrizable manifold, and $f: M \rightarrow \mathbb{R}$ be a locally Lipschitz function. Suppose that $F \subset O$ be respectively a closed and an open subset of $T^{*} M$, such that $F_{x}=F \cap T_{x}^{*} M$ is convex for each $x \in M$, and $d_{x} f \in F_{x}$ for almost every $x$ in $\operatorname{dom}(d f)$. If $\epsilon: M \rightarrow(0,+\infty)$ is a continuous function, then there exists a $C^{\infty}$ function $g: M \rightarrow \mathbb{R}$ such that $\left(x, d_{x} g\right) \in O$ and $|f(x)-g(x)|<\epsilon(x)$, for each $x \in M$.

We need a couple of lemmas.

Lemma 8.2 Under the hypothesis of the theorem, for each $x_{0} \in M$ and each neighborhood $N$ of $x_{0}$, we can find a closed set $C$ and a relatively compact open subset $W$ of $T^{*} M$ with $x_{0} \in V=\pi_{*}(W) \subset N, C \subset \bar{W} \subset O, C \cap \pi_{*}^{-1}(V) \subset W$. Moreover, the intersections $C \cap T_{x}^{*} M$ and $\bar{W} \cap T_{x}^{*} M$ are convex for each $x \in M$, and $d_{x} f \in C \cap T_{x}^{*} M$, for almost every $x \in V$.

Proof. This is essentially a local result at $x_{0}$, so we can assume that $M$ is an open subset of $\mathbb{R}^{k}$, hence $T^{*} M=M \times\left(\mathbb{R}^{k}\right)^{*}$. We will use the usual Euclidean norm $\|\cdot\|_{2}$ on $\mathbb{R}^{k}$. 
Choose a compact neighborhood $K \subset N$ of $x_{0}$, then $f$ is a Lipschitz function on a neighborhood of $K$. It follows that $\kappa=\sup \left\{\left\|d_{x} f\right\|_{2} \mid x \in \operatorname{dom}(f) \cap K\right\}$ is finite. Let us consider the decreasing sequence of sets $S_{n}=\bar{D}_{n}$, where

$$
D_{n}=\left\{d_{y} f \mid y \in \operatorname{dom}(f), d_{y} f \in F_{y},\left\|y-x_{0}\right\|_{2} \leq 1 / n\right\}
$$

Choose $n_{0}$ such that the Euclidean ball $\left\{y \in \mathbb{R}^{k} \mid\left\|y-x_{0}\right\|_{2} \leq 1 / n_{0}\right\}$ is contained in $K$. Then $S_{n}$ is compact for $n \geq n_{0}$. The intersection $S_{\infty}=\bigcap_{n \geq 0} S_{n}$ is compact and contained in the closed convex set $F_{x_{0}} \subset\left(\mathbb{R}^{k}\right)^{*}$, we can therefore find a pair of open convex subsets $A, B$ in $\left(\mathbb{R}^{k}\right)^{*}$, with $\bar{B}$ compact, such that $S_{\infty} \subset A \subset \bar{A} \subset B$, and $\left\{x_{0}\right\} \times \bar{B} \subset O$. Since $\bar{B}$ is compact, this implies that $\left\{y \in \mathbb{R}^{k} \mid\left\|y-x_{0}\right\| \leq 1 / n\right\} \times \bar{B} \subset O$, for $n$ large enough. Since $A$ is open and contains $S_{\infty}$ the intersection of the decreasing sequence of compact sets $S_{n}, n \geq 0$, we must have $S_{n} \subset A$, for a large enough $n$. It is now obvious that we can take $C=\left\{y \in \mathbb{R}^{k} \mid\left\|y-x_{0}\right\| \leq 1 / n\right\} \times \bar{A}$ and $W=\left\{y \in \mathbb{R}^{k} \mid\left\|y-x_{0}\right\| \leq 1 / n\right\} \times B$ for $n$ large enough.

Lemma 8.3 Let $U$ be an open subset of $\mathbb{R}^{k}$, and $f: U \rightarrow \mathbb{R}$ is a locally Lipschitz function. Suppose that $F \subset O$ are respectively a closed and open subset of $T^{*} U=$ $U \times\left(\mathbb{R}^{k}\right)^{*}$, with $F_{x}=\left\{p \in \mathbb{R}^{k} \mid(x, p) \in F\right\}$ convex for each $x \in U$. If $\left(x, d_{x} f\right) \in F$, for almost every $x \in U$, then for each open subset $U^{\prime}$, such that its closure $\overline{U^{\prime}}$ is compact and contained in $U$, and each $\epsilon>0$, there exists a $C^{\infty}$ function $g: U^{\prime} \rightarrow \mathbb{R}$ such that $\left(x, d_{x} g\right) \in O$ and $|f(x)-g(x)|<\epsilon$, for each $x \in U^{\prime}$.

Proof. In this proof we will denote by $\|x\|_{2}$ the usual Euclidean norm of $x \in \mathbb{R}^{k}$.

Since $\overline{U^{\prime}}$ is compact and contained in $U$, using lemma 8.2 above, or more precisely its proof, we can find a family $\left(V_{i}\right)_{i \in I}$ of open subsets of $\mathbb{R}^{k}$, and a family $\left(B_{i}\right)_{i} \in I$ of open relatively compact convex subsets of $\left(\mathbb{R}^{k}\right)^{*}$, such that $\overline{U^{\prime}} \subset \bigcup_{i \in I} V_{i} \subset U, \bigcup_{i \in I} \bar{V}_{i} \times \bar{B}_{i} \subset O$, and $d_{z} f \in B_{i}$, for almost every $z \in$ $V_{i} \cap \operatorname{dom}(d f)$. Since $\overline{U^{\prime}}$ is compact and covered by the family of open sets $\left(V_{i}\right)_{i \in I}$, we can find $\delta_{0}>0$ such that, for each $x \in \overline{U^{\prime}}$, the Euclidean ball $\bar{B}\left(x, \delta_{0}\right)=\{z \in$ $\left.\mathbb{R}^{k} \mid\left\|z-x_{0}\right\|_{2} \leq \delta_{0}\right\}$ is contained in $V_{i}$ for some $i \in I$.

We will now use a convolution argument. Let $\left(\rho_{\delta}\right)_{\delta>0}$ be a family of functions $\rho_{\delta}: \mathbb{R}^{k} \rightarrow[0,+\infty)$ of class $C^{\infty}$ with $\rho_{\delta}=0$, if $\|y\|_{2} \geq \delta$, and $\int_{\mathbb{R}^{k}} \rho_{\delta}(y) d y=1$.

When $\delta<\delta_{0}$, the convolution $f_{\delta}(x)=\int_{\mathbb{R}^{k}} \rho_{\delta}(y) f(x-y) d y$ makes sense for $x$ in a neighborhood of $\overline{U^{\prime}}$. As is well know $f_{\delta}$ is of class $C^{\infty}$ on a neighborhood of $\overline{U^{\prime}}$, moreover $f_{\delta}$ converges to $f$ uniformly on $\overline{U^{\prime}}$ when $\delta \rightarrow 0$. Because $f$ is locally Lipschitzian, for $x \in \overline{U^{\prime}}$, the derivative $d_{x} f_{\delta}$ is equal to $\int_{\mathbb{R}^{k}} \rho_{\delta}(y) d_{x-y} f d y$. For such an $x \in \overline{U^{\prime}}$, we can choose $i \in I$ such that $\bar{B}\left(x, \delta_{0}\right) \subset V_{i}$. Since $\rho_{\delta}(y) \geq 0$ is zero for $\|y\|_{2} \geq \delta, \int_{\mathbb{R}^{k}} \rho_{\delta}(y) d y=1$, and $d_{x} f$ is in the convex set $B_{i}$, for almost every $z \in V_{i} \cap \operatorname{dom}(d f)$, we see that $d_{x} f_{\delta}$ is in $\bar{B}_{i}$. Hence $\left(x, d_{x} f_{\delta}\right) \in \bar{V}_{i} \times \bar{B}_{i} \subset O$. Since $f_{\delta}$ converges to $f$ uniformly on $\overline{U^{\prime}}$ when $\delta \rightarrow 0$, we can take $g=f_{\delta}$ for $\delta>0$ small enough to have $\sup _{x \in \bar{V}}\left|f_{\delta}(x)-f(x)\right|<\epsilon$. 
Proof of theorem 8.1. We can assume $M$ connected (if not, we can just prove the theorem for each connected component of $M$ and then "glue up" things). In particular, since $M$ is metrizable, it is $\sigma$-compact and from every open cover of $M$ we can extract a countable subcover. We can then tacitly assume that every open cover we use is countable. Using lemma 8.2 , we can find a family $\left(W_{n}\right)_{n \in \mathbb{N}}$ of open relatively compact subsets, and a family $C_{n}$ of compact subsets of $T^{*} M$ satisfying the following conditions

(i) for each $n \in \mathbb{N}$, the open subset $V_{n}=\pi_{*}\left(W_{n}\right)$ of $M$ is contained in the domain of a smooth $C^{\infty}$ chart of $M$, and $M=\bigcup_{n \in \mathbb{N}} V_{n}$,

(ii) for each $n \in \mathbb{N}$, the closure $\bar{W}_{n}$ is compact and contained in $O$. Moreover $C_{n} \subset \bar{W}_{n}, C_{n} \cap \pi^{-1}\left(V_{n}\right) \subset W_{n}$,

(iii) for each $n \in \mathbb{N}$ and each $x \in V_{n}$, the intersections $T_{x}^{*} M \cap \bar{W}_{n}, T_{x}^{*} M \cap C_{n}$ are convex, and

(iv) for every $n \in \mathbb{N}$ and for almost $x \in V_{n} \cap \operatorname{dom}(d f)$, we have $\left(x, d_{x} f\right) \in C_{n}$.

The family $\left(V_{n}\right)_{n \in \mathbb{N}}$ is an open covering of the metric space $M$, therefore we can find a locally finite open cover $\left(V_{n}^{\prime}\right)_{n \in \mathbb{N}}$ of $M$ with $V_{n}^{\prime} \subset V_{n}$.

By standard topological methods, see for example 3.2 page 167 in [7], we can find an open cover $\left(U_{m}\right)_{m \in \mathbb{N}}$ of $M$ such that

(v) for each $x \in M$, there exists $n \in \mathbb{N}$ such that $\bigcup_{x \in U_{m}} U_{m} \subset V_{n}^{\prime}$.

In particular, each of the sets $U_{m}$ is contained in some $V_{n}$, it is therefore relatively compact.

We now fix $\left(\varphi_{m}\right)_{m \in \mathbb{N}}$, a $C^{\infty}$ partition of unity on $M$ subordinated to the cover $\left(U_{m}\right)_{m \in \mathbb{N}}$. The support of $\varphi_{m}$ is compact since it is contained in $U_{m}$, so $K_{m}=\sup _{x \in M}\left\|d_{x} \varphi_{m}\right\|<+\infty$.

Since $W_{n}$ is compact and contained in the open set $O$, we can find $\epsilon_{n}^{1}>0$ such that if $(x, p) \in \bar{W}_{n}$ and $p^{\prime} \in T_{x}^{*} M$ satisfy $\left\|p^{\prime}-p\right\| \leq \epsilon_{n}^{1}$ then $\left(x, p^{\prime}\right) \in O$. By the compactness of the closure $\bar{V}_{n}$, and the continuity of the (strictly) positive function $\epsilon$, we can find $\epsilon_{n}^{2}>0$ such that $\epsilon(x)>\epsilon_{n}^{2}$, for each $x \in V_{n}$. We set $\epsilon_{n}=\min \left(\epsilon_{n}^{1}, \epsilon_{n}^{2}\right)>0$.

Since $\bar{U}_{m}$ is compact and the cover $\left(V_{n}^{\prime}\right)_{n \in \mathbb{N}}$ is locally finite, the set $J_{m}=$ $\left\{n \in \mathbb{N} \mid \bar{U} m \subset V_{n}^{\prime}\right\}$ is finite (and not empty by (v)). Therefore we can find $\eta_{m}>0$ such that $\eta_{m} \leq \epsilon_{n}$ and $K_{m} \eta_{m} \leq \epsilon_{n} / 2^{m+1}$, for each $n \in J_{m}$. We define the open sets $W_{m}^{\prime \prime}=\bigcap_{n \in J_{m}} W_{n}$, the compact sets $C_{m}^{\prime \prime}=\bigcap_{n \in J_{m}} C_{n}$ and $V_{m}^{\prime \prime}=$ $\bigcap_{n \in J_{m}} V_{n}^{\prime}$. We have $\bar{U}_{m} \subset V_{m}^{\prime \prime}$. Moreover, from condition (iii) and (iv), we obtain the following two properties

(vi) for each $m \in \mathbb{N}$, we have $C_{m}^{\prime \prime} \cap \pi_{*}^{-1}\left(V_{m}^{\prime \prime}\right) \subset W_{m}^{\prime \prime} \cap \pi_{*}^{-1}\left(V_{m}^{\prime \prime}\right)$, and for each $m \in \mathbb{N}$ and each $x \in V_{m}^{\prime \prime}$, the intersection $T_{x}^{*} M \cap C_{m}^{\prime \prime}$ is convex,

(vii) for every $m \in \mathbb{N}$ and for almost every $x \in V^{\prime \prime} \cap \operatorname{dom}(f)$, we have $\left(x, d_{x} f\right) \in C_{m}^{\prime \prime}$.

Since $V_{m}^{\prime \prime}$ is contained in some $V_{n}$, which is contained in a domain of a chart, and we can apply lemma 8.3 with $U=V_{m}^{\prime \prime}, U^{\prime}=U_{m}, O=W_{n}^{\prime \prime} \cap \pi_{*}^{-1}\left(V_{m}^{\prime \prime}\right)$, and $F=C_{m}^{\prime \prime} \cap \pi_{*}^{-1}\left(V_{m}^{\prime \prime}\right)$ we find $g_{m}$ defined and $C^{\infty}$ on $U_{m}$ such that

(viii) for each $x \in U_{m}$, we have $\left|g_{m}(x)-f(x)\right| \leq \eta_{m}$, and $\left(x, d_{x} g_{m}\right) \in W_{m}^{\prime \prime}$.

We now show that the $C^{\infty}$ function $g=\sum_{i \in \mathbb{N}} \varphi_{i} g_{i}$ does satisfy the conclusion of the theorem. For this we fix $x \in M$, and $L_{x}=\left\{m \mid x \in U_{m}\right\}$. By condition 
$(v)$ above, we can choose $n$ such that $\bigcup_{m \in L_{x}} \bar{U}_{m} \subset V_{n}^{\prime}$. By the choice of the $\eta_{m}$, it follows that $\eta_{m} \leq \epsilon_{n}$ and $K_{m} \eta_{m} \leq \epsilon_{n} / 2^{m+1}$, for each $m \in L_{x}$. Moreover, from (viii), we obtain $\left|g_{m}(x)-f(x)\right|<\eta_{m} \leq \epsilon_{n}$, and $\left(x, d_{x} g_{m}\right) \in W_{n}$, for $m \in L_{x}$. Now $g(x)=\sum_{m \in L_{x}} \varphi_{m}(x) g_{m}(x)$ and $\sum_{m \in L_{x}} \varphi_{m}(x)=1$. It follows that

$$
|g(x)-f(x)| \leq \sum_{m \in L_{x}} \varphi_{m}(x)\left|g_{m}(x)-f(x)\right| \leq \sum_{m \in L_{x}} \varphi_{m}(x) \epsilon_{n}<\epsilon .
$$

For the derivative, we observe that

$$
d_{x} g=\sum_{x \in L_{x}} \varphi_{m}(x) d_{x} g_{m}+\sum_{x \in L_{x}} g_{m}(x) d_{x} \varphi_{m} .
$$

The first term of this sum belongs to the convex set $\bar{W}_{n} \cap T_{x}^{*} M$, since $d_{x} g_{m} \in$ $W_{m}^{\prime \prime} \cap T_{x}^{*} M$, for $m \in L_{x}$, and $W_{m}^{\prime \prime} \subset W_{n}$, for $m \in L_{x}$. By the choice of $\epsilon_{n}$, it suffices to show that the second term $\sum_{m \in L_{x}} g_{m}(x) d_{x} \varphi_{m}$ has a norm bounded by $\epsilon_{n}$. In fact, we have $\sum_{m \in L_{x}} \varphi_{m}(y)=1$, for each $y$ in a neighborhood of $x$, hence $\sum_{m \in L_{x}} d_{x} \varphi_{m}=0$. Multiplying this equality by $f(x)$ gives $\sum_{m \in L_{x}} f(x) d_{x} \varphi_{m}=0$. Therefore we obtain

$$
\begin{aligned}
\left\|\sum_{m \in L_{x}} g_{m}(x) d_{x} \varphi_{m}\right\| & =\left\|\sum_{x \in L_{x}}\left(g_{m}(x)-f(x)\right) d_{x} \varphi_{m}\right\| \\
& \leq \sum_{x \in L_{x}}\left|g_{m}(x)-f(x)\right|\left\|d_{x} \varphi_{m}\right\| \\
& \leq \sum_{m \in L_{x}} \eta_{m} K_{m} \leq \sum_{m \in L_{x}} \epsilon_{n} / 2^{m+1} \leq \epsilon_{n}
\end{aligned}
$$

We add a comment to clarify things for people knowing Nonsmooth Analysis, see [4]. The following proposition is well know, see [4] pages $62-63$, we provide a slightly different proof.

Proposition 8.4 Under the hypothesis of theorem 8.1, for each $x \in \operatorname{dom}(d f)$, we do have $\left(x, d_{x} f\right) \in F$.

Proof. The statement is local in nature, so we can assume $M$ is an open set in $\mathbb{R}^{k}$, and $f$ is Lipschitzian on $M$. This implies that $\kappa=\sup \left\{\left\|d_{x} f\right\|_{2} \mid x \in \operatorname{dom}(d f)\right\}$ is finite. We can replace $F \subset T^{*} M=M \times\left(\mathbb{R}^{k}\right)^{*}$ by $F \cap M \times\left\{p \in \mathbb{R}^{k} \mid\|p\|_{2} \leq \kappa\right\}$. Hence we can assume $F \cap K \times\left(\mathbb{R}^{k}\right)^{*}$ is compact for each compact subset $K$ of $M$. Let $O_{n}$ be a decreasing sequence of open relatively compact subsets of $M \times\left(\mathbb{R}^{k}\right)^{*}$, with $\bigcap_{n \in \mathbb{N}} O_{n}=F \cap K \times\left(\mathbb{R}^{k}\right)^{*}$. Using lemma 8.3, we can find a sequence $g_{n}$ of $C^{\infty}$ maps defined on a neighborhood $K$ of $x$ such that $\left(y, d_{y} g_{n}\right) \in O_{n}$, for each $n \in \mathbb{N}$, and each $y \in K$, and $\sup _{y \in K}\left|f(y)-g_{n}(y)\right| \leq 1 / n^{2}$. Fix $v \in \mathbb{R}^{k}$. For $n$ large enough, we have $x+n^{-1} v \in K$, therefore $n \mid f\left(x+n^{-1} v\right)-f(x)-\left(g_{n}\left(x+n^{-1} v\right)-\right.$ $\left.g_{n}(x)\right) \mid \leq 2 n^{-1}$. Since $x \in \operatorname{dom}(d f)$, we have $d_{x} f(v)=\lim _{n \rightarrow \infty} n\left[f\left(x+n^{-1} v\right)-\right.$ $f(x)]$. By the mean value theorem, there exists $y_{n}$ in the segment $\left[x, x+n^{-1} v\right]$ 
such that $n\left(g_{n}\left(x+n^{-1} v\right)-g_{n}(x)\right)=d_{y_{n}} g_{n}(v)$. Since $y_{n}$ converges to $x$, and $\left(y_{n}, d_{y_{n}} g_{n}\right) \in O_{n} \subset O_{1}$ which is a relatively compact subset, we can extract a subsequence converging to some $\left(x, p_{v}\right)$. Because $\bigcap_{n \in \mathbb{N}} O_{n} \subset F$, we obtain $p_{v} \in F_{x}$. Hence, we obtained that for each $v \in \mathbb{R}^{k}$, there exists a $p_{v} \in F_{x}$, with $d_{x} f(v)=p_{v}(v)$, since $F_{x}$ is convex, an application of Hahn-Banach theorem gives $d_{x} f \in F_{x}$.

It follows from this proposition that there is a closed smallest set $F \subset M$ such that $F_{x}$ is convex for each $x \in M$, and $\left(x, d_{x} f\right) \in F$, for almost every $x \in M$. This set is obtained in the following way, we take $D^{*} f$ the closure in $T^{*} M$ of $\left\{\left(x, d_{x} f\right) \mid x \in \operatorname{dom}(d f)\right\}$. The set $D^{*} f(x)=D^{*} f \cap T_{x}^{*} M$ is compact, hence by Carathéodory's theorem its convex hull $\partial f(x)$ in $T_{x}^{*} M$ is also compact. This set $\partial f(x)$ is the generalized Clarke derivative at $x$, see [4] page $61-62$. The closed set we are looking for is $\partial f=\bigcup_{x \in M} \partial f(x) \subset T^{*} M$, the graph of the multivalued map $x \mapsto \partial f(x)$. Of course, knowing that, it suffices to take in theorem 8.1 the set $\partial f$ for $C$. However, we stated theorem 8.1 as it will usually be used.

The following theorem is a consequence of theorem 8.1.

Theorem 8.5 Suppose $H: T^{*} M \rightarrow \mathbb{R}$ is continuous and convex in each fiber $T_{x}^{*} M, x \in M$. If $u: M \rightarrow \mathbb{R}$ is locally Lipschitz, with its derivative satisfying $H\left(x, d_{x} u\right) \leq c$ almost everywhere, then for each $\epsilon>0$, there exists a $C^{\infty}$ function $u_{\epsilon}: M \rightarrow \mathbb{R}$ such that $H\left(x, d_{x} u\right) \leq c+\epsilon$ and $\left|u(x)-u_{\epsilon}(x)\right| \leq \epsilon$, for each $x \in M$.

Proof. This is clearly a consequence of theorem 8.1 with $F=\left\{(x, p) \in T^{*} M\right.$ $H(x, p) \leq c\}$ and $O=\left\{(x, p) \in T^{*} M \mid H(x, p) \leq c+\epsilon\right\}$.

Notice that in theorem 8.5 above, we do not assume that $H$ is superlinear or even coercive, hence it does cover the case of the evolution inequality $\partial_{t} u(x, t)+$ $H\left(t, x, \partial_{x} u(x, t)\right) \leq c$ almost everywhere, as soon as $H(t, x, p)$ is continuous in $(t, x, p)$ and convex in $p$.

\section{References}

[1] M. BARDI and I. CAPPUZZO-DOLCETA, Optimal Control and Viscosity Solutions of Hamilton-Jacobi-Bellman Equations, Systems 83 Control: Foundations and Applications, Birkhäuser Boston Inc., Boston, MA, 1997.

[2] G. BARLES, Solutions de viscosité des équations de Hamilton-Jacobi, Mathématiques et Applications, 17, Springer-Verlag, Paris, 1994.

[3] G. BUTTAZO, M. GIAQUINTA and S. HILDEBRANDT, One-dimensional variational problems, an introduction, Oxford Lecture Series in Mathematics and its Applications, 15, Oxford University Press, Oxford, 1998.

[4] F. H. CLARKE, Optimization and Nonsmooth Analysis, Canadian Mathematical Society Series of Monographs and Avanced Texts, John Wiley \& Sons, New York, 1983. 
[5] G. CONTRERAS, Action potential and weak KAM solutions. Calc. Var. Partial Differ. Equ. 13(4) (2001), 427-458.

[6] G. CONTRERAS, R. ITURRIAGA, G. PATERNAIN and M. PATERNAIN, Lagrangian graphs, minimizing measures and Mañé's critical values. Geom. Funct. Anal. 8(5) (1998), 788-809.

[7] J. DUGUNDJI, Topology, Allyn and Bacon Inc., Boston, 1970.

[8] L. C. EVANS, Partial differential equations, Graduate studies in Mathematics, 19, American Mathematical Society, Providence, RI, 1998.

[9] A. FATHI, Théorème KAM faible et théorie de Mather sur les systèmes lagrangiens. C. R. Acad. Sci. Paris, Série I 324 (1997), 1043-1046.

[10] A. FATHI, Solutions KAM faibles conjuguées et barrières de Peierls, $C$. $R$. Acad. Sci. Paris, Série I 325 (1997), 649-652.

[11] A. FATHI, Weak KAM Theorem in Lagrangian Dynamics, book to appear, Cambridge University Press.

[12] P. L. LIONS, G. PAPANICOLAU and S. R. S. VARADHAN, Homogenization of Hamilton-Jacobi equation, unpublished preprint, 1987.

[13] E. MADERNA, Invariance of global solutions of the Hamilton-Jacobi equation. Bull. Soc. Math. Fr. 130(4) (2002), 493-506.

[14] R. MAÑÉ, Global variational methods in conservative dynamics, $18^{\circ}$ Colóquio Brasileiro de Matemática, IMPA, Rio de Janeiro.

[15] J. MATHER, Action minimizing invariant measures for positive definite Lagrangian systems. Math. Z. 207 (1991), 169-207.

[16] J. MATHER, Variational construction of connecting orbits. Ann. Ins. Fourier 43 (1993), 1349-1386.

[17] G. PATERNAIN and M. PATERNAIN, Critical values of autonomous Lagrangian systems. Comment. Math. Helvetici 72 (1997), 481-499.

[18] J. P. PIER, Amenable locally compact groups, Pure and applied mathematics, John Wiley \& Sons, New York, 1984.

Received 7 November 2002; accepted 20 February 2003

Published Online First 13 August 2007 